Voix et Images

voixetimages

\title{
BIBLIOGRAPHIE DE JACQUES POULIN
}

\section{Tania Grégoire}

Volume 45, numéro 2 (134), hiver 2020

Jacques Poulin

URI : https://id.erudit.org/iderudit/1069514ar

DOI : https://doi.org/10.7202/1069514ar

Aller au sommaire du numéro

\section{Éditeur(s)}

Université du Québec à Montréal

\section{ISSN}

0318-9201 (imprimé)

1705-933X (numérique)

Découvrir la revue

\section{Citer ce document}

Grégoire, T. (2020). BIBLIOGRAPHIE DE JACQUES POULIN. Voix et Images, 45(2),

107-135. https://doi.org/10.7202/1069514ar d'utilisation que vous pouvez consulter en ligne.

https://apropos.erudit.org/fr/usagers/politique-dutilisation/ 


\section{B IBLIOGR A PHIE DE JACQUES POULIN}

$$
++
$$

\section{TANIA GRÉGOIRE}

Université de Moncton

\section{E U V R ES}

\section{A. Rom a n s}

+ Mon cheval pour un royaume, Montréal, Éditions du Jour, coll. «Les romanciers du jour», 1967, 119 p. (Réédition: Montréal, Leméac, coll. «Poche. Québec», 1987, 192 p.)

+ Jimmy, Montréal, Éditions du Jour, coll. «Les romanciers du jour», 1969, 158 p. (Rééditions: Montréal, Leméac, coll. «Roman québécois», 1978, 172 p.; Montréal, Stanké, coll. «Roman 10/10», 1985, 165 p. ; Montréal/Arles, Leméac/Actes Sud, coll. «Babel», 1999 et 2009, 180 p. ; Montréal, Leméac, coll. «Nomades», 2016, 180 p.)

+ Le cœur de la baleine bleue, Montréal, Éditions du Jour, coll. «Les romanciers du jour», 1970, 200 p. (Rééditions: Montréal, Éditions du Jour, coll. «Le petit jour», 1979, 200 p.; Montréal, Leméac, coll. «Poche. Québec», 1987, 200 p. ; Montréal, Bibliothèque québécoise, coll. «Littérature. BQ», 1994 et 2012, 162 p.)

+ Faites de beaux rêves, Montréal, L'Actuelle, 1974, 163 p. (Rééditions: Montréal, Bibliothèque québécoise, coll. «Littérature. BQ», 1988 et 2003, 200 p.)

+ Les grandes marées, Montréal, Leméac, coll. «Roman québécois», 1978, 200 p. (Rééditions: Montréal, Leméac, coll. «Poche. Québec», 1986, 200 p. ; Montréal, Bibliothèque québécoise, coll. «Littérature. BQ», 1990, 209 p. ; Montréal/Arles, Leméac/Actes Sud, coll. «Babel», 1995, 213 p. ; Montréal, Leméac, coll. «Nomades», 2015, 208 p.)

+ Volkswagen Blues, Montréal, Québec/Amérique, coll. «Littérature d'Amérique», 1984, 290 p. (Rééditions: Montréal, Québec loisirs, 1985, 290 p.; Montréal, Québec/Amérique, coll. «Espace», 1987, 290 p. ; Paris, Éditions Jean Picollec, 1988, 301 p.; Montréal/Arles, Leméac/ Actes Sud, coll. «Babel», 1998, 323 p.; Montréal, Leméac, coll. «Roman», 1999, 295 p.; Montréal, Leméac, coll. «Nomades», 2015, 323 p.)

+ Le vieux Chagrin, Montréal/Arles, Leméac/Actes Sud, 1989, 155 p. (Rééditions: Montréal/ Arles, Leméac/Actes Sud, coll. «Babel», 1995 et 2008, 187 p.; Montréal, Leméac, coll. «Nomades», 2016, 187 p.)

+ La tournée d'automne, Montréal, Leméac, coll. «Roman», 1993, 208 p. (Rééditions: Montréal/ Arles, Leméac/Actes Sud, coll. «Babel», 1996, 190 p.; Montréal, Leméac, coll. «Nomades», 2016, 190 p.)

+ Chat sauvage, Montréal/Arles, Leméac/Actes Sud, 1998, 188 p. (Rééditions: Montréal/Arles, Leméac/Actes Sud, coll. «Babel», 2000, 224 p.; Montréal, Leméac, coll. «Nomades», 2016, 224 p.) 
+ Les yeux bleus de Mistassini, Montréal/Arles, Leméac/Actes Sud, 2002, 187 p. (Rééditions: Montréal/Arles, Leméac/Actes Sud, coll. «Babel», 2011, 199 p. ; Montréal, Leméac, coll. «Nomades», 2015, 199 p.)

+ La traduction est une histoire d'amour, Montréal/Arles, Leméac/Actes Sud, 2006, 131 p. (Réédition: La traduction est une histoire d'amour suivi de L'anglais n'est pas une langue magique, Montréal, Leméac, coll. «Nomades», 2019, 268 p.)

+ L'anglais n'est pas une langue magique, Montréal/Arles, Leméac/Actes Sud, 2009, 155 p.

+ L’homme de la Saskatchewan, Montréal/Arles, Leméac/Actes Sud, 2011, 120 p.

+ Un jukebox dans la tête, Montréal, Leméac, 2015, 146 p.

\section{B. Textes de création dans des périodiques et des anthologies}

+ «Texte inédit», Nord, nº 2, hiver 1972, p. 30-37.

+ «Le Journal de la Grande Sauterelle», Études françaises, vol. XXI, n 3, hiver 1985, p. 103-106.

+ «Le sergent Kate», Premier amour (collectif), Montréal, Stanké, coll. «10/10», 1988, p. 187-189.

+ «Un coup de fil de Jack», Lettres québécoises, n 83, automne 1996, p. 7.

\section{Entretiens}

+ BELLEMARE, Madeleine, "Le livre du mois: Volkswagen Blues (entrevue)», Nos livres, vol. XV, juin-juillet 1984, p. 4-5.

+ CLOUTIER, Rachel, GIGNAC, Rodrigue, Vincent NADEAU et Richard PLAMONDON, «Entrevue avec Jacques Poulin», Nord, n² 2, hiver 1972, p. 9-29.

+ DORION, Gilles et Cécile DUBÉ, «Entrevue. Jacques Poulin», Québec français, n 34, mai 1979, p. 33-35. Article reproduit dans Romanciers du Québec. Hubert Aquin, Roch Carrier, Jacques Ferron, Jacques Godbout, Anne Hébert, André Langevin, Jacques Poulin, Gabrielle Roy, FélixAntoine Savard, Yves Thériault, Québec, Québec français, 1980, p. 147-154.

+ LAPOINTE, Jean-Pierre et Yves THOMAS, «Entretien avec Jacques Poulin», Voix et Images, vol. XV, nº 1, automne 1989, p. 8-14.

+ LÉVESQUE, Robert, «“Bon qu'à ça." Entretien avec Jacques Poulin», Liberté, vol. L, nº 4, décembre 2009, p. 96-103.

+ MAJOR, André, «Entrevues. Un poète et un romancier», Le Devoir, 8 juin 1967, p. 6.

+ OUELLET, François, «Jacques Poulin», Nuit blanche, n 45, septembre-octobre-novembre 1991, p. $40-43$.

+ ROY, Michelle et Jean GAUTHIER, «Conversation avec Jacques Poulin», La revue Pantoute, n³, septembre-octobre-novembre 1980, p. 22-23.

+ TREMBLAY, Yolaine, «Rencontre avec Jacques Poulin», Azimuth, décembre 1976, s. p.

+ VASSEUR, François et Michelle ROY, «Voyage à travers l'Amérique. Entrevue avec Jacques Poulin", Nuit blanche, nº 14, juin-juillet-août 1984, p. 50-52.

+ VIGNEAULT, Benny, «Jacques Poulin: trouver le traducteur en nous», Les libraires, 10 avril 2006, en ligne: https://revue.leslibraires.ca/entrevues/litterature-quebecoise/jacques-poulintrouver-le-traducteur-en-nous (page consultée le 8 novembre 2019).

+ YOKEN, Mel B., «Jacques Poulin», Entretiens québécois, t. I, Montréal, Pierre Tisseyre, 1986, p. 160-171. 


\section{EUVRES PARUES EN TRADUCTION}

+ «My Horse for a Kingdom», The Jimmy Trilogy, traduction anglaise de Sheila Fischman, Toronto, House of Anansi Press, 1979, p. 9-58.

+ "Jimmy», The Jimmy Trilogy, traduction anglaise de Sheila Fischman, Toronto, House of Anansi Press, 1979, p. 59-156.

+ «The Heart of the Blue Whale», The Jimmy Trilogy, traduction anglaise de Sheila Fischman, Toronto, House of Anansi Press, 1979, p. 157-250.

+ Spring Tides, traduction anglaise de Sheila Fischman, Toronto, House of Anansi Press, 1986, 166 p. (Réédition: Brooklyn, Archipelago Books, 2007, 237 p.)

+ Volkswagen Blues, traduction anglaise de Sheila Fischman, Toronto, McClelland and Stewart, 1988, 213 p. (Réédition: Toronto, Cormorant Books, 2004, 222 p.)

+ Mr. Blue, traduction anglaise de Sheila Fischman, Montréal, Véhicule Press, 1993, 160 p. (Réédition: Brooklyn, Archipelago Books, 2012, 150 p.)

+ Volkswagen Blues, traduction italienne de Maria Rosa Baldi, Rome, Hortus Conclusus, 2000, $304 \mathrm{p}$.

+ Autumn Rounds, traduction anglaise de Sheila Fischman, Toronto, Cormorant Books, 2002, $164 \mathrm{p}$.

+ Wild Cat, traduction anglaise de Sheila Fischman, Toronto, Cormorant Books, 2003, 178 p.

+ Volkswagen Blues, traduction espagnole d'Antonio Marquez, Barcelone, Plaza \& Janés, 2003, $257 \mathrm{p}$.

+ Le cour de la baleine bleue, traduction arabe, Damas, Édition Al-Hassad, 2006, 192 p.

+ My Sister's Blue Eyes, traduction anglaise de Sheila Fischman, Toronto, Cormorant Books, 2007, $171 \mathrm{p}$.

+ Translation is a Love Affair, traduction anglaise de Sheila Fischman, Brooklyn, Archipelago Books, 2009, $144 \mathrm{p}$.

+ Volkswagen Blues, traduction roumaine de Denisa-Adriana Oprea, Cluj-Napoca (Roumanie), Limes, 2010, $236 \mathrm{p}$.

+ English is Not a Magic Language, traduction anglaise de Sheila Fischman, Montréal, Véhicule Press, 2016, $140 \mathrm{p}$.

\section{I I. PR I X E T D IS T INCTIO N S}

+ Prix de La Presse 1974, Faites de beaux rêves.

+ Prix du Gouverneur général 1978, Les grandes marées.

+ Prix Canada-Belgique 1984, Volkswagen Blues.

+ Prix Québec-Paris 1989, Le vieux Chagrin.

+ Prix Molson du roman 1990, Le vieux Chagrin.

+ Prix Louis-Hémon 1990, Le vieux Chagrin.

+ Prix France-Québec-Jean-Hamelin 1991, Le vieux Chagrin.

+ Prix Athanase-David 1995, ensemble de l'œuvre.

+ Prix d'excellence des arts et de la culture 1999, Chat sauvage.

+ Prix Molson des arts du Canada 2000, ensemble de l'œuvre.

+ Prix littéraire des collégiens 2003, Les yeux bleus de Mistassini.

+ Prix d'excellence des arts et de la culture 2003, décerné par le Salon international du livre de Québec, Les yeux bleus de Mistassini.

+ Prix des lecteurs d'Herblay 2008, La traduction est une histoire d'amour. 
+ Prix Gilles-Corbeil 2008, ensemble de l'œuvre.

\section{RÉCEPTION CRITIQUE ET PRINCIPALES ÉTUDES}

\section{A. Monographies}

1. Ouvrages consacrés à Poulin

+ BAYLE PETRELLI, Françoise, Pour une lecture de Le vieux Chagrin de Jacques Poulin, Dolianova (CA), Edizioni Grafica del Parteolla, 1993, 120 p.

+ HÉBERT, Pierre, Jacques Poulin. La création d'un espace amoureux, Ottawa, Presses de l'Université d'Ottawa, coll. «Euvres et auteurs», 1997, 205 p.

+ L'ITALIEN-SAVARD, Isabelle, Isabelle L'Italien-Savard présente Les grandes marées de Jacques Poulin, Montréal, Leméac, coll. «Parallèle», 2000, 76 p.

+ MIRAGLIA, Anne Marie, L'écriture de l'Autre chez Jacques Poulin, Candiac, Éditions Balzac, coll. «L'univers des discours», 1993, 245 p.

+ SOCKEN, Paul G., The Myth of the Lost Paradise in the Novels of Jacques Poulin, Londres/ Toronto, Associated University Presses, 1993, 126 p.

\section{Ouvrages partiellement consacrés à Poulin}

+ ACQUISTO, Joseph, Crusoes and Other Castaways in Modern French Literature. Solitary Adventures, Newark, University of Delaware Press, 2012, 282 p.

+ ALLARD, Jacques, Le roman du Québec. Histoire, perspectives, lectures, Montréal, Québec/ Amérique, 2000, $446 \mathrm{p}$.

+ BAYLE PETRELLI, Françoise, Deçà et delà l'Atlantique: deux romanciers intimistes, CabanisPoulin, Cagliari, Società Poligrafica Sarda, 1994, 147 p.

+ BOIVIN, Aurélien, Pour une lecture du roman québécois. De Maria Chapdelaine à Volkswagen Blues, Québec, Nuit blanche éditeur, coll. «Littérature», 1996, 365 p.

+ CHAPMAN, Rosemary, Sitting the Quebec Novel. The Representation of Space in Francophone Writing in Quebec, Bern, Peter Lang, 2000, 282 p.

+ CHASSAY, Jean-François, L'ambiguité américaine. Le roman québécois face aux États-Unis, Montréal, XYZ éditeur, coll. «Théorie et littérature», 1995, 198 p.

+ DUSSAULT-FRENETTE, Catherine, L'expression du désir au féminin dans quatre romans québécois contemporains, Montréal, Nota bene, coll. «Convergences», 2015, 167 p.

+ GAUVIN, Lise, Langagement. L'écrivain et la langue au Québec, Montréal, Boréal, 2000, 254 p.

+ HAREL, Simon, Le voleur de parcours. Identité et cosmopolitisme dans la littérature québécoise contemporaine, Montréal, Le Préambule, coll. «L'univers des discours», 1989, $309 \mathrm{p}$.

+ HUGGAN, Graham, Territorial Disputes. Maps and Mapping Strategies in Contemporary Canadian and Australian Fiction, Toronto, University of Toronto Press, coll. «Theory/Culture», 1994, $198 \mathrm{p}$.

+ LAMONTAGNE, André, Le roman québécois contemporain. Les voix sous les mots, Montréal, Fides, coll. «Nouvelles études québécoises», 2004, 288 p.

+ LINTVELT, Jaap, Aspects de la narration. Thématique, idéologie et identité. Guy de Maupassant, Julien Green, Anne Hébert, Jacques Poulin, Québec/Paris, Nota bene/L’Harmattan, coll. «Littérature(s)», 2000, 306 p.

+ MAGNAN, Lucie-Marie et Christian MORIN, Lectures du postmodernisme dans le roman québécois, Québec, Nuit blanche éditeur, coll. «Littérature(s)», 1997, 220 p. 
+ MARCATO FAlZONI, Franca, Canada ieri e oggi, Fasano (Italie), Schena Editore, coll. «Biblioteca della Ricerca, Cultura Straniera», 1986, s. p.

+ MARCOTTE, Gilles, Écrire à Montréal, Montréal, Boréal, coll. «Papiers collés», 1997, 186 p.

+ MORENCY, Jean, Le mythe américain dans les fictions d'Amérique, de Washington Irving à Jacques Poulin, Québec, Nuit blanche éditeur, coll. «Terre américaine», 1994, 258 p.

+ NEPVEU, Pierre, L'écologie du réel. Mort et naissance de la littérature québécoise contemporaine, Montréal, Boréal, coll. «Papiers collés», 1988, 248 p.

+ NEPVEU, Pierre, Lectures des lieux, Montréal, Boréal, coll. «Papiers collés», 2004, 272 p.

+ PATERSON, Janet M., Figures de l'Autre dans le roman québécois, Québec, Nota bene, coll. «Littérature», 2004, 487 p.

+ PRZYCHODZEN, Janusz, De la simplicité comme mode d'emploi. Le minimalisme en littérature québécoise, Québec, Presses de l'Université Laval, 2014, 194 p.

+ SHEK, Ben-Zion, French-Canadian and Québécois Novels, Toronto, Oxford University Press, 1991, $160 \mathrm{p}$.

+ SING, Pamela V., Villages imaginaires: Édouard Montpetit, Jacques Ferron et Jacques Poulin, Montréal, Fides/CÉTUQ, coll. «Nouvelles études québécoises», 1995, 272 p.

+ SIROIS, Antoine, Lecture mythocritique du roman québécois. Anne Hébert, Jacques Ferron, Jacques Poulin, Gabrielle Roy, Yves Thériault, Montréal, Triptyque, 1999, 132 p.

+ THIBEAUlT, Jimmy, Des identités mouvantes. Se définir dans le contexte de la mondialisation, Montréal, Nota bene, coll. «Terre américaine», 2015, 393 p.

+ TREMBLAY, Roseline, L'écrivain imaginaire. Essai sur le roman québécois (1960-1995), Montréal, Hurtubise HMH, coll. «Cahiers du Québec. Littérature», 2004, 606 p.

+ UNTERBERG, Jens, Fremde und Feinde. Phänomenologie des Heterogenen im Quebecer Roman, Berlin, De Gruyter, 234 p.

\section{B. Études}

+ AMOSSI, Ruth, «Les modalités du récit symbolique dans Les grandes marées de Jacques Poulin», Danielle Schaub, Janice Kulyk Keefer et Richard E. Sherwin (dir.), Precarious Present/ Promising Future? Ethnicity and Identities in Canadian Literature, Jérusalem, The Hebrew University Magnes Press, coll. «Canadian Studies», 1996, p. 145-159.

+ ANOLL, Lidia, «Le vieux Chagrin, de Jacques Poulin, ou le pouvoir d'un fantastique merveilleux», Francofonia, n 7, 1998, p. 9-28.

+ ANOLL, Lidia, «Québec de l'écriture, Québec du cœur: un regard porté sur l'œuvre de Jacques Poulin», Anna Paola Mossetto et Jean-François Plamondon (dir.), Lectures de Québec, actes du colloque du Centro Interuniversitario di Studi Quebecchesi tenu à Turin du 27 au 29 février 2008, Bologne, Pendragon, 2009, p. 157-173.

+ BAKER, Rachel, «Transidentitaire: sexe, genre et sexualité dans Volkswagen Blues de Jacques Poulin et Le goût des jeunes filles de Dany Laferrière», The Arbutus Review, vol. V, nº 1 , automne 2014, p. 191-215.

+ BASTIEN, Sophie, «Les grandes marées, dans le roman de Jacques Poulin: phénomène naturel ou courant culturel?», Canadian Literature, nº 198, 2008, p. 48-56.

+ BASTIEN, Sophie, «Dire l'émotion en ne la disant pas: la manière de Jacques Poulin", Anny Krzyanowskiej et Renaty Jakubczuk (dir.), Parler des émotions: entre langue et littérature, Lublin (Pologne), Wydawnictwo Uniwersytetu Marii Curie-Sklodowskiej, 2011, p. 205-214. 
+ BAYLE PETRELLI, Françoise, "Le refus de la ville dans les derniers romans de Jacques Poulin», Canada e Italia verso il duemila: metropoli a confronto, actes du $9^{\mathrm{e}}$ Convegno Internazionale di Studi Canadesi, Milano, Schena editore, coll. «Biblioteca Della Ricerca, Cultura Straniera», 1992, p. 171-201.

+ BAYLE PETRELLI, Françoise, «De l'essentiel à l'anecdotique. L'influence de J. Poulin sur l'œuvre de Savoie», Études. Revue de culture contemporaine, n 37, 1994, p. 395-406.

+ BEAUDOIN, Réjean et André LAMONTAGNE, «La littérature canadienne de langue française», Revue francophone de Louisiane, vol. VII, n 2, automne 1993, p. 145-182.

+ BEAUDOIN, Réjean et André LAMONTAGNE, «Un demi-siècle de réception critique de la littérature québécoise au Canada anglais: 1939-1989», Denis Saint-Jacques (dir.), Tendances actuelles en histoire littéraire canadienne, Québec, Nota bene, coll. «Les Cahiers du Centre de recherche en littérature québécoise», 2003, p. 85-105.

+ BERERHI, Afifa, «Le vieux Chagrin de Jacques Poulin», Bouba Mohammedi Tabti (coordonnatrice), Littérature canadienne. Impressions de lecture, Blida (Algérie), Éditions du Tell, 2005, p. 103-109.

+ BERNOVSKY, Victor, «Les héros de Jacques Poulin sur la route américaine», Interculturel francophonies, n 32, novembre-décembre 2017, p. 199-219.

+ BIRON, Michel, François DUMONT et Élisabeth NARDOUT-LAFARGE, «Jacques Poulin et le roman en mode mineur», Histoire de la littérature québécoise, avec la collaboration de MartineEmmanuelle Lapointe, Montréal, Boréal, 2007, p. 544-551.

+ BISTODEAU, Linda, «Quelques aspects de la mimesis géographique dans Volkswagen Blues de Jacques Poulin», Gregorio C. Martin (dir.), Selected Proceedings of the Pennsylvania Foreign Language Conference (1991-1992), Pittsburgh, Duquesne University, 1995, p. 54-59.

+ BOISCLAIR, Isabelle, "Masculinité et maternage dans Le vieux Chagrin de Jacques Poulin», Voix et Images, vol. XXXII, nº 2, hiver 2007, p. 49-61.

+ BOIVIN, Aurélien, "La tournée d'automne ou comment renaître par l'amour», Québec français, $\mathrm{n}^{\circ}$ 125, printemps 2002, p. 88-90.

+ BOIVIN, Aurélien, "Le vieux Chagrin ou une leçon d'écriture», Québec français, n 138, été 2005, p. 94-97.

+ BOIVIN, Aurélien, "Les grandes marées ou un regard troublant sur la société contemporaine», Québec français, n 149, printemps 2008, p. 105-107.

+ BONSIGNORE, Giacomo, «Jacques Poulin: une conception de l'écriture», Études françaises, vol. XXI, nº 3, hiver 1985, p. 19-26.

+ BOUÉ, Pilar Andrade, «Poétique de l'intertextualité dans les romans de Jacques Poulin», Cuadernos de investigacion filologica, vol. XXIX-XXX, 2003-2004, p. 235-248.

+ BOULESTEIX, Caroline, "Recherche d'identité par le voyage dans Volkswagen Blues de Jacques Poulin », Dana Puiu (dir.), Identity and Alterity in Canadian Literature/Identité et altérité dans la littérature canadienne, Cluj-Napoca (Roumanie)/Baia Mare, Risoprint/Canadian Studies Center/ Centre d'études canadiennes, 2003, p. 25-28.

+ BOURQUE, Paul-André, «La fascination de l'enfance, de la tendresse et de la mort chez Jacques Poulin ou la recherche de l'androgyne absolue», Nord, n² 2, hiver 1972, p. 74-92.

+ BOURQUE, Paul-André, «Jacques Poulin et la mer», Melvin Gallant (dir.), Mer et littérature, actes du colloque international sur «La mer dans les littératures d'expression française du xx siècle» tenu à Moncton les 22-23-24 août 1991, Moncton, Éditions d'Acadie, 1992, p. 313-325. 
+ BRIÈRE, Éloïse A., «Ventriloquizing the Native. Whose Voice Is It?», The French Review, vol. LXXXIV, nº 6, mai 2011, p. 1200-1212.

+ CHASSAY, Jean-François, «Topographie du lecteur (explicite) dans le roman québécois contemporain", La Licorne, n² 27, 1993, p. 105-117.

+ CHOQUETTE, Sylvie, «L'archétype du temps circulaire chez Ernest Hemingway et Jacques Poulin", Études littéraires, vol. VIII, nº 1, 1975, p. 43-55.

+ CLÉMENT, Anne-Marie, "La narrativité à l'épreuve de la discontinuité», René Audet et Andrée Mercier (dir.), La narrativité contemporaine au Québec, t. I: La littérature et ses enjeux narratifs, avec la collaboration de Denise Cliche, Québec, Presses de l'Université Laval, 2004, p. 107-135.

+ CÔTÉ, Jean-Denis, «Volkswagen Blues a quinze ans!», Études. Revue de culture contemporaine, $\mathrm{n}^{\circ} 46,1999$, p. 77-92.

+ CUBEDDU, Stefania, «La ville de Québec dans l'espace imaginaire de Jacques Poulin », Hélène Amrit, Anna Giaufret-Harvey et Sergio Zoppi (dir.), Regards sur la littérature québécoise.

Hommage à Gaston Miron, actes du troisième colloque de l'Association des jeunes chercheurs européens en littérature québécoise, Rome, Bulzoni, coll. "Centro per lo studio delle letterature e delle culture delle aree emergenti», 2001, p. 59-65.

+ CUBEDDU, Stefania, «Les grandes marées de Jacques Poulin: un échiquier fictif... dans le paradis d'une île», Africa America Asia Australia, n 23, Rome, Bulzoni Editore, 2002, p. 43-50.

+ CUBEDDU, Stefania, "Repousser les frontières pour rentrer chez soi: enquête sur la littérature québécoise contemporaine», Muriel Rouyer, Catherine De Wrangel, Emmanuelle Bousquet et Stefania Cubeddu (dir.), Regards sur le cosmopolitisme européen: frontières et identités, Bruxelles, Peter Lang, coll. «Europe des cultures», 2011, p. 369-387.

+ CUBEDDU, Stefania, «Jacques Poulin et Italo Calvino. Prolégomènes pour une lecture croisée de la ville en littérature», Beïda Chikhi et Anne Douaire-Banny (dir.), Villes, vies, visions. Les villes, propriétés de l'écrivain, actes du colloque international organisé par le Centre international d'études francophones de l'Université Paris-Sorbonne à Abou Dhabi, Paris, L'Harmattan, coll. «Espaces littéraires», 2012, p. 89-101.

+ CUBEDDU, Stefania, «La ville invisible de Jacques Poulin. Une lecture de la ville de l'auteur québécois à travers le regard d'Italo Calvino", Anne Douaire-Banny (dir.), Isthmes francophones: du texte aux chants du monde. Mélanges offerts à Beïda Chikhi, Paris, Presses de l'Université Paris-Sorbonne, coll. «Lettres francophones», 2012, p. 393-404.

+ CUBEDDU, Stefania, «Enquête aux marges de la ville. Le Québec de Jacques Poulin», AnneYvonne Julien (dir.), Littératures québécoise et acadienne contemporaines. Au prisme de la ville, avec la collaboration d'André Magord, Rennes, Presses universitaires de Rennes, coll. «Plurial», 2014, p. 281-290.

+ DEITZ, Ritt, "Au bord de l'américanité: la fonction du voyage dans Volkswagen Blues», Iris. Journal of French Critical Studies, vol. V, n 2, 1991, p. 87-93.

+ DEMERS, Jeanne, «Besoin de tendresse, over ou Jacques Poulin conteur», Études françaises, vol. XXI, n 3, hiver 1985, p. 27-35.

+ DEN TOONDER, Jeanette, «Une littérature nord-américaine de langue française», Rachel Killick (dir.), Uncertain Relations: Some Configurations of the "Third Space" in Francophone Writings of the Americas and of Europe, Oxford, Peter Lang, 2005, p. 49-62.

+ DESMEULES, Georges, "Le tennis au service de Jacques Poulin», Québec français, n 114, été 1999, p. 80-82. 
+ DION, Robert, «Let's Talk English Here. Les représentations de l'anglais dans Copies conformes et Volkswagen Blues», Barbara Buchenau et Annette Paatz (dir.), Do the Americas Have a Common Literary History?, Francfort/Berlin/Berne/Bruxelles/New York/Oxford/Vienne, Peter Lang, coll. «Interamericana», 2002, p. 427-447.

+ DOLBEC, Nathalie, «D'un tableau l'autre: le parcours de l'ekphrasis dans Volkswagen Blues de Jacques Poulin", Canadian Literature, n 184, printemps 2005, p. 27-43.

+ DOLBEC, Nathalie, «Les contours du descriptif dans Volkswagen Blues de Jacques Poulin», Canadian Literature, n 216, printemps 2013, p. 85-99.

+ DORION, Gilles, «L'espace et ses trajets psychologiques», Québec français, nº 109, printemps 1988, p. 66-69.

+ DORION, Gilles, «Une chronique du mensonge», Chantal Adobati, Maria Aldouri-Lauber et Emanuela Hager (dir.), Wenn Ränder Mitte werden. Zivilisation, Literatur und Sprache im interkulturellen Kontext. Festschrift für F. Peter Kirsch zum 60. Geburtstag, Vienne, WUV, 2001, p. 213-225.

+ DUPUIS, Gilles, «Poétique de la dérive dans les romans de Jacques Poulin», Francofonia. Studi e Ricerche Sulle Letterature di Lingua Francese, vol. XXXIV, printemps 1998, p. 41-68.

+ DUPUIS, Gilles, «De l'eutopie à la dystopie. Utopies croisées dans Les grandes marées de Poulin», Dix-huitième siècle, n 34, 2002, p. 165-173.

+ DUPUIS, Gilles, «Du mot juste au mot rare. Dictionnaires et glossaires dans l'œuvre d'Hubert Aquin et Jacques Poulin», Gerardo Aceranza (dir.), Dictionnaires français et littératures québécoise et canadienne-française, Ottawa, Éditions David, coll. «Voix savantes», 2005, s. p.

+ DUPUIS, Gilles, «Jacques Poulin, romancier de la dérive», Études québécoises, nº 1, 2007, p. 25-42.

+ DUPUIS, Gilles, «L'île aux chats. Incursion dans l'archipel félin de Jacques Poulin», Carmelina Imbroscio, Nadia Minerva et Patrizia Oppici (dir.), Flottements autour du thème insulaire en hommage à Carminella Biondi, Berne, Peter Lang, coll. «Franco-Italica», 2008, p. 449-456.

+ DUPUIS, Gilles, «La redécouverte de Québec par Maillet et Jacques Poulin», Klaus Dieter Ertler et Martin Löschnigg (dir.), Inventing Canada/Inventer le Canada, Frankfurt am Main, Peter Lang, coll. "Canadiana», 2008, p. 43-56.

+ ERTLER, Klaus-Dieter, «L'optique dans le dialogue interculturel: Les grandes marées et Volkswagen Blues de Jacques Poulin», Cahiers francophones d'Europe centre-orientale, n ${ }^{\circ} 6$, 1995, p. 301-313.

+ ERTLER, Klaus-Dieter, «VII. Postmoderne und "écritures migrantes". Die achtziger und neunziger Jahre», Kleine Geschichte des franko-kanadischen Romans, Tübingen, Gunter Narr Verlag, coll. «Narr studienbücher», 2000, p. 209-230.

+ FALZONI, Franca Marcato, «Storia di una lingua divenuta impotente. Les grandes marées di Jacques Poulin», actes du $6^{\mathrm{e}}$ Convegno internazionale di studi canadesi, Fasano, Schena editore, 1985, p. 263-280.

+ FASANO, Linda, «Introduction à l'étude de Volkswagen Blues», Giovanni Dotoli, Il Canada del nuovo secolo. Gli archivi della memoria, actes du Convegno internazionale tenu à Monopoli du 30 mai au 3 juin 2001, Fasano, Schena editore, 2002, p. 383-400.

+ FASANO, Linda, «Le dernier Poulin. Voyages pour un retour au pays natal», Giovanni Dotoli (dir.), Culture e letterature di lingua francese in Canada, actes du Seminario internazionale tenu à Monopoli les 17, 18 et 19 mai 2004, Fasano, Schena editore, 2005, p. 203-214. 
+ FASANO, Linda, «La route de la fable. "Le chat botté" de Charles Perrault dans Jimmy de Poulin», Giovanni Dotoli (dir.), Canada. Le rotte della libertà, actes du Convegno Internazionale tenu à Monopoli du 5 au 9 octobre 2005, Fasano, Schena editore, 2006, p. 213-220.

+ FASANO, Linda, «Tre personaggi in uno: Poulin», Rivista di studi canadesi/Canadian Studies Review/Revue d'études canadiennes, nº 20, 2007, p. 143-171.

+ FASANO, Linda, "La ville dans l'œuvre de Poulin», Angela Buono et Marina Zito, Ambiente e società canadesi/Environnement et sociétés canadiennes/Environment and Canadian societies, Napoli, L'Orientale, 2010, p. 265-280.

+ FILION, Pierre, «La marche des mots. Propos-contacts», Études françaises, vol. XXI, nº 3 , hiver 1985, p. 97-102.

+ FINELL, Susanna, "The United States in the Literary Imagination of the Quebec Novel», Revue francophone de Louisiane, vol. II, nº 2, hiver 1987, p. 30-37.

+ FRATTA, Carla, "Vivre dans les îles dystopiques. Poulin, Alberto Moravia et Giovanni Papini», Gilles Dupuis et Dominique Garand (dir.), Italie-Québec. Croisements et coïncidences littéraires, Québec, Nota bene, 2009, p. 111-121.

+ GALLAYS, François, «L'iconographie dans Volkswagen Blues de Jacques Poulin», Diffractions. Romans et nouvelles du Québec, Orléans, Éditions David, 2000, p. 211-227.

+ GARNEAU, Jacques, "Le cœur de la baleine bleue ou le labyrinthe intérieur», Nord, n 2 , hiver 1972, p. 59-68.

+ GASQUY-RESCH, Yannick, «Chapitre 4. Dire l'Amérique», Yannick Gasquy-Resch (dir.), Littérature du Québec, Vanves, ÉDICEF, coll. «Histoire littéraire de la francophonie. Universités francophones», 1994, p. 243-250.

+ GAUVIN, Lise, «L'en-deçà des voyages: exploration de quelques romans québécois», Franca Marcato-Falzoni (dir.), La deriva delle francofonie, t. IV : Autour de l'univers souterrain dans la littérature québécoise. Atti di seminari annuali di Letterature Francophone. Centro Interfacoltà Sorella Clarke dell' Università di Bologna, Bagni di Lucca, 4-5 maggio 1989, Bologne, Éditrice CLUEB Bologna, coll. «Bussola», 1990, p. 225-241.

+ GAUVIN, Lise, «Littérature québécoise et francophonie: un statut particulier», La Licorne, $n^{\circ} 27$, automne 1993, p. 417-426.

+ GAUVIN, Lise, «Glissements de langues et poétiques romanesques: Poulin, Ducharme, Chamoiseau ", Littérature, no 101, février 1996, p. 5-24.

+ GAUVIN, Lise, «Écriture, surconscience et plurilinguisme: une poétique de l'errance», Christiane Albert (dir.), Francophonie et identités culturelles, Paris, Karthala, coll. «Lettres du Sud», 1999, p. 13-29.

+ GAUVIN, Lise, «Le palimpseste poulinien: réécritures, emprunts, autotextualités», Romanica Silesiana, $\mathrm{n}^{\circ}$ 2, 2007, p. 190-203.

+ GEORGESCU, Delia, «Les gens et les marées», Central European Journal of Canadian Studies/ Revue d'études canadiennes en Europe centrale, n 7, 2011, p. 53-64.

+ GIRAUDO, José Eduardo, «Mnémosyne en fourgonnette ou la poéthique de la mémoire: sur Volkswagen Blues, de Jacques Poulin», Cadernos do IL, nº 9, janvier 1993, p. 49-56.

+ GODIN, Jean-Cléo, «Entre la pierre et l'extase», Nord, n 2, hiver 1972, p. 38-47.

+ GONTARD, Marc, «Littérature et altérité dans Kamouraska d’Anne Hébert et Volkswagen Blues de Jacques Poulin", Marc Gontard et Maryse Bray (dir.), Regards sur la francophonie, Rennes, Presses universitaires de Rennes, coll. «Plurial», 1996, p. 315-322. 
+ GREILICH, Susanne, «Durchmessungen und Vermessungen des nordamerikanischen Raumes in Poulins Volkswagen Blues», Robert Dion, Ute Fendler, Albert Gouaffo et Christoph Vatter (dir.), Interkulturelle Kommunikation in der frankophonen Welt. Literatur, Medien, Kulturtransfer. Festschrift zum 60. Geburtstag von Hans-Jürgen Lüsebrink/La communication interculturelle dans le monde francophone. Transferts culturels, littéraires et médiatiques. Mélanges offerts à HansJürgen Lüsebrink à l'occasion de son 60 anniversaire, Ingbert, Röhrig, 2012, p. 81-93.

+ GRUBER, Iris, «L'autoréférentialité et le postmoderne dans Les grandes marées et Le désert mauve», Canadian studies in Europe/Études canadiennes en Europe, 2002, p. 63-74.

+ GUENTHER, Beatrice, «Land and Cityscape in Lise Gauvin's and Jacques Poulin's Narratives: Between Cultural Memory and L'invention du quotidien », Magali Compan et Katarzyna Pieprzak (dir.), Land and Landscape in Francographic Literature: Remapping Uncertain Territories, Newcastle, Cambridge Scholars, 2007, p. 60-87.

+ HAREL, Simon, «La tentation cosmopolite», Voix et Images, vol. XIV, nº 2, hiver 1989, p. 281-293.

+ HÉBERT, Pierre, «Jacques Poulin: de la représentation de l'espace à l'espace de la représentation", Études françaises, vol. XXI, n³ 3, hiver 1985, p. 37-53.

+ HODGSON, Richard et Ralph SARKONAK, «Deux hors-la-loi québécois: Jacques Godbout et Jacques Poulin », Québec Studies, n 8, avril 1989, p. 27-36.

+ HOTTE, Lucie, «Transtextualité anglo-américaine. Volskwagen Blues de Jacques Poulin et L'écureuil noir de Daniel Poliquin", Lise Gauvin, Cécile Van Den Avenne, Véronique Corinus et Ching Selao (dir.), Littératures francophones. Parodies, pastiches, réécritures, Lyon, ENS Éditions, coll. «Signes», 2013, p. 105-116.

+ HUE, Bernard, «Légendes et chansons du vieux monde dans le roman canadien d'expression française», Bernard Hue (dir.), Métissage du texte. Bretagne, Maghreb, Québec, Rennes, Presses universitaires de Rennes/Centre d'études des littératures et civilisations francophones, coll. «Plurial», 1993, p. 271-280.

+ HYMAN, Roger, «Writing Against Knowing, Writing Against Certainty; or What's Really Under the Veranda in Jacques Poulin's Volkswagen Blues », Journal of Canadian Studies/Revue d'études canadiennes, vol. XXXIV, n 3, automne 1999, p. 106-133.

+ JARQUE, Alexandra, "Sur les traces de la lectrice dans Le vieux Chagrin de Jacques Poulin», Québec Studies, nº 18, avril 1994, p. 138-148.

+ KÁDÁR, Krisztina, «Discours de la modernité et de la tradition dans Volkswagen Blues de Poulin", Cahiers francophones d'Europe centre-orientale. Revue annuelle de pluriculturalisme, $n^{\circ} 10,2000$, p. 307-317.

+ KAPOLKA, Karolina, "Le voyage en quête d'identité. Volkswagen Blues de Poulin», Krzysztof Jarosz, Les images de l'Amérique dans les littératures en langues romanes, Katowice, Wydawnictwo Uniwersytetu Slaskiego, 2005, p. 158-169.

+ KLAUS, Peter, «Les écrivains francophones du Canada se réapproprient leur histoire et leur territoire?», Klaus-Dieter Ertler et Martin Löschnigg (dir.), Inventing Canada/Inventer le Canada, Frankfurt am Main, coll. «Canadiana», Peter Lang, 2008, p. 57-64.

+ KVIA, K., "Apprendre le bonheur. Une étude de La tournée d'automne de Jacques Poulin", Tribune, $\mathrm{n}^{\circ} 10,1999$, p. 39-55.

+ KWATERKO, Jozef, «Urbi et orbi. La ville proche et lointaine dans le roman québécois», Lucie K. Morisset, Luc Noppen et Denis Saint-Jacques (dir.), Ville imaginaire, ville identitaire: échos de Québec, Québec, Nota bene, 1999, p. 317-326. 
+ LABONTÉ, René, «Québec-Californie: la Californie à travers la fiction littéraire québécoise», The French Review, vol. LXII, n 5, avril 1989, p. 803-814.

+ LABRIE, Gilles, "Quebec's Quiet Revolution in Jacques Poulin's Les grandes marées", The French Review, vol. LXXIX, n 5, avril 2006, p. 1037-1048.

+ LACHANCE, Maurice, «Une (ou deux) voix dans l'orchestre», Études françaises, vol. XXI, n 3, hiver 1985, p. 55-65.

+ LAMONTAGNE, André, «L'autre Poulin», TTR. Traduction, terminologie, rédaction, vol. XV, nº 1, 2002, p. 45-61.

+ LAPOINTE, Jean-Pierre, «Sur la piste américaine. Le statut des références littéraires dans l'œuvre de Jacques Poulin», Voix et Images, vol. XV, n 1, automne 1989, p. 15-27.

+ LAPOINTE, Jean-Pierre, «Narcisse travesti. L'altérité des sexes chez trois romanciers québécois contemporains», Voix et Images, vol. XVIII, n 1, automne 1992, p. 11-25.

+ LAPOINTE, Jean-Pierre, «L'américanité du roman québécois contemporain: altérité exotique ou endotique», Études canadiennes/Canadian Studies, n 33, décembre 1992, p. 289-297.

+ LAVOIE, Carlo, «Maurice Richard: du joueur à la figure», RSSI. Recherches sémiotiques/ Semiotics Inquiry, vol. XXII, nos 1-3, 2002, p. 211-228.

+ LEAHY, David, «Running in the Family, Volkswagen Blues and Heroine: Three Post/Colonial Post-Modernist Quests?», Kunapipi, vol. XIV, n³ 3, 1992, p. 67-82.

+ LEBLANC, Julie, «Le système pictural de Volkswagen Blues de Jacques Poulin», Jean-Michel Lacroix, Simone Vauthier et Héliane Ventura (dir.), Image et récit: littérature(s) et arts visuels du Canada, Paris, Presses de la Sorbonne Nouvelle, 1993, p. 195-208.

+ LEDUC, Mario, «Le bonheur autrement. L’héritage décrié de Robinson Crusoé dans Les grandes marées de Jacques Poulin», Voix et Images, vol. XXVI, nº 3, printemps 2001, p. 569-584.

+ LEMELIN, Jean-Marc, "Quatre pistes de lecture de Volkswagen Blues», Mœebius, n 57 , automne 1993, p. 101-116.

+ LEVASSEUR, Jean, "L'ambiguïté temporelle dans Jimmy de Jacques Poulin», Les Cahiers de l'APFUCC, série 3, nº 2, 1989, p. 39-52.

+ LEVASSEUR, Jean, «La quête des racines par l'exil: étude comparée de De quoi t'ennuies-tu, Evvelyne? de Gabrielle Roy et de Volkswagen Blues de Jacques Poulin», Marie-Lyne Piccione (dir.), Un pays, une voix, Gabrielle Roy, Talence, La maison des sciences de l'homme d'Aquitaine/ Centre d'études canadiennes de Bordeaux, 1991, p. 37-46.

+ LEVASSEUR, Jean, «Du manuscrit à l'édition princeps. L'histoire d'une grande marée chez Jacques Poulin: la figure du patron", Studies in Canadian Literature/Études en littérature canadienne, vol. XVII, nº 1, 1992, p. 52-61.

+ LEVASSEUR, Jean, «L'évolution du symbole féminin dans la narratologie de Jacques Poulin (1967-1984)», Raija Koski, Kathleen Kells et Louise Forsyth (dir.), Les discours féminins dans la littérature postmoderne au Québec, Lewinston, The Edwin Mellen Press, 1993, p. 307-315.

+ L’HÉRAULT, Pierre, "Volkswagen Blues: traverser les identités», Voix et Images, vol. XV, nº 1 , automne 1989, p. 28-42.

+ LINDBERG, Svante, «La langue-culture face au monde dans trois romans québécois (20072009)", Cécilia W. Francis et Robert Viau (dir.), Trajectoires et dérives de la littérature-monde. Poétiques de la relation et du divers dans les espaces francophones, Amsterdam, Rodopi-Brill, coll. «Francopolyphonies», 2013, p. 499-517.

+ LINTVELT, Jaap, «Le double thématique et narratif dans Le vieux Chagrin de Jacques Poulin», Dalhousie French Studies, vol. XXIII, automne-hiver 1992, p. 87-96. 
+ LINTVELT, Jaap, «La dualité identitaire dans l'œuvre de Jacques Poulin», Rapports. Het Franse boek, vol. LXVI, $\mathrm{n}^{\text {os }} 1-2,1996$, p. 22-30.

+ LINTVELT, Jaap, «Jacques Poulin's Novels: From Duality to Fusion of Identity», Tity De Vries (dir.), Dynamics of Modernization: European-American Comparisons and Perceptions, Amsterdam, VU UP, 1998, p. 135-145.

+ LINTVELT, Jaap, «Le double identitaire et narratif dans les romans de Jacques Poulin», Jaap Lintvelt, Richard Saint-Gelais, W. M. Verhoeven et Catherine Raffi-Béroud (dir.), Roman contemporain et identité culturelle en Amérique du Nord/Contemporary Fiction and Cultural Identity in North America, Québec, Nota bene, coll. «Littérature(s)», 1998, p. 59-81.

+ LINTVELT, Jaap, «L'espace identitaire de la ville de Québec dans le roman québécois depuis 1960 », Lucie K. Morisset, Luc Noppen et Denis Saint-Jacques (dir.), Ville imaginaire, ville identitaire: échos de Québec, Québec, Nota bene, 1999, p. 295-316.

+ LINTVELT, Jaap, «Le voyage identitaire aux États-Unis dans le roman québécois», Jean Morency, Jeanette Den Toonder et Jaap Lintvelt (dir.), Romans de la route et voyages identitaires, Québec, Nota bene, coll. «Terre américaine», 2006, p. 55-88.

+ LÜSEBRINK, Hans-Jürgen, «Frontera de cristal/Gläserne Grenze: Carlos Fuentes (Mexiko) und Jacques Poulin (Québec) als interkulturelle Grenzgänger», Wolfgang Brücher (dir.), Grenzverschiebungen: Interdisziplinäre Beiträge zu einem zeitlosen Phänomen. Internationales Symposium des interdisziplinären Forschungsschwerpunktes «Grenzregionen und Interferenzräume» der Philosophischen Fakultäten der Universität des Saarlandes in Saarbrücken und Forbach 10.-12. Mai 2001, St. Ingbert (Allemagne), Röhrig Universitätsverlag, coll. «Annales Universitatis Saraviensis/Philosophische Fakultät», 2003, p. 147-164.

+ LÜSEBRINK, Hans-Jürgen, «Die Faszination der Bücher. Zu dem Roman Les yeux bleus de Mistassini (2002) von Poulin (Québec)», Michael Einfalt, Ottmar Ette, Ursula Erzgräber et Franziska Sick, Intellektuelle Redlichkeit/Intégrité intellectuelle. Literatur, Geschichte, Kultur. Festschrift für Joseph Jurt, 2005, p. 373-383.

+ LÜSEBRINK, Hans-Jürgen, «"Lost in Translation”. Übersetzung und Exilerfahrung bei Eva Hoffmann (Polen/Kanada/USA) und Poulin (Québec, Kanada)», Alberto Gil et Manfred Schmeling (dir.), Kultur übersetzen. Zur Wissenschaft des Übersetzens im deutsch-französischen Dialog/Traduire la culture. Le dialogue franco-allemand et la traduction, Berlin, Akad.-Verl., 2009, p. 97-106.

+ LÜSEBRINK, Hans-Jürgen, «Éloge du français et apologie de la lecture. L'anglais n'est pas une langue magique de Jacques Poulin», Gilles Dupuis et Klaus-Dieter Ertler (dir.), À la carte. Le roman québécois (2005-2010), Berne, Peter Lang, 2011, p. 335-348.

+ MACFARLANE, Heather, «Volkswagen Blues Twenty-Five Years Later. Revisiting Poulin's Pitsémine», Studies in Canadian Literature/Études en littérature canadienne, vol. XXXIV, nº 2, 2009, p. 5-21.

+ MAILHOT, Laurent, "Bibliothèques imaginaires. Le livre dans quelques romans québécois», Études françaises, vol. XVIII, nº 3, hiver 1982, p. 81-92.

+ MAILHOT, Laurent, «Volkswagen Blues, de Jacques Poulin, et autres "histoires américaines" du Québec», Ouvrir le livre. Essais, Montréal, l’Hexagone, coll. «Essais littéraires», 1992, p. 299-309.

+ MAINDRON, André, «Regards sur le fleuve: de Caron et de Poulin», Études canadiennes/ Canadian Studies. Revue interdisciplinaire des études canadiennes en France, n 50, juin 2001, p. 189-198. 
+ MAINGUY, Maude, «Le sport dans l'œuvre de Jacques Poulin», Québec français, nº 157, printemps 2010, p. 34-35.

+ MARCOTTE, Gilles, «Histoires de zouaves», Études françaises, vol. XXI, n³ 3, hiver 1985, p. 7-17.

+ MARTONYI, Éva, "Le rêve américain dans Volkswagen Blues de Jacques Poulin», Fritz Peter Kirsch et Waldemar Zacharasiewicz (dir.), Perspectives interculturelles, Wien, Centre d'études canadiennes, 2001, p. 159-167.

+ MELIC, Katarina, «Volkswagen Blues de Jacques Poulin. Visions de l'Amérique et cartographie identitaire», Jelena Novakovic et Vladimir Gvozden (dir.), Modern Canada. Prejudices, stereotypes, authenticity/Le Canada moderne. Préjugés, stéréotypes, authenticité, Beograd, Megatrend University, 2013, p. 173-182.

+ MÉVEL, Pierre-Alexis et Dawn M. CORNELIO, «Collision and Collusion: Contrasting Representations of the Translator-Author Relationship in Two Contemporary Francophone Novels», TTR. Traduction, terminologie, rédaction, vol. XXIX, n 1, $1^{\mathrm{er}}$ semestre 2016, p. 139-160.

+ MICHAUD, Ginette, "Récits postmodernes?», Études françaises, vol. XXI, n³ 3, hiver 1985, p. 67-88.

+ MICHAUD, Ginette, «Jacques Poulin: petit éloge de la lecture ralentie», François Gallays, Sylvain Simard et Robert Vigneault (dir.), Le roman contemporain au Québec (1960-1985), Montréal, Fides/Centre de recherche en civilisation canadienne-française de l'Université d'Ottawa, coll. «Archives des lettres canadiennes», 1992, p. 363-380.

+ MICHON, Jacques, «Heureux qui comme Ulysse», Voix et Images, vol. XI, nº 1, automne 1985, p. 135-139.

+ MILOT, Louise et Fernand ROY, «Des Mille et une nuits au Vieux Chagrin», Louise Milot et Jaap Lintvelt (dir.), Le roman québécois depuis 1960. Méthodes et analyses, Québec, Presses de l’Université Laval/Centre de recherche en littérature québécoise, 1992, p. 119-132.

+ MIRAGLIA, Anne Marie, "Lecture, écriture et intertextualité dans Volkswagen Blues", Voix et Images, vol. XV, $\mathrm{n}^{\circ} 1$, automne 1989, p. 51-57.

+ MiRAGLIA, Anne Marie, "L'Amérique et l'américanité chez Jacques Poulin», Urgences, n 34, décembre 1991, p. 34-45.

+ MIRAGLIA, Anne Marie, "Le récit de voyage en quête de l'Amérique», Dalhousie French Studies, vol. XXIII, automne-hiver 1992, p. 29-34.

+ MIRAGLIA, Anne Marie, «Texts engendering Texts: A Québécois Rewriting of American Novels», Elaine D. Cancalon et Antoine Spacagna (dir.), Intertextuality in Literature and Film, Gainesville, University Press of Florida, 1994, p. 49-60.

+ MIRAGLIA, Anne Marie, «Le lecteur fictif et la lecture critique chez Jacques Poulin. Du Vieux Chagrin à Chat sauvage", Québec Studies, nº 29, avril 2000, p. 104-114.

+ MODENESI, Marco, «Jacques Poulin et Jack Waterman. L'écrivain et ses doubles», Alain Rey, Pierre Brunel, Philippe Desan et Jean Pruvost (dir.), De l'ordre et de l'aventure. Langue, littérature, francophonie. Hommage à Giovanni Dotoli, Paris, Hermann, 2014, p. 459-467.

+ MORENCY, Jean, «Le cœur de la baleine bleue de Jacques Poulin: de la poésie au roman», Urgences, $\mathrm{n}^{\circ}$ 28, 1990, p. 30-40.

+ MORENCY, Jean, «La thématique de la mer et la structuration de l'œuvre romanesque de Jacques Poulin», Melvin Gallant (dir.), Mer et littérature, actes du colloque international sur «La mer dans les littératures d'expression française du xx siècle» tenu à Moncton les 22-23-24 août 1991, Moncton, Éditions d'Acadie, 1992, p. 327-336. 
+ MORENCY, Jean, «Mythes et mythologies de l'Amérique dans le roman québécois contemporain. Analyse comparée des romans de Beaulieu et de Jacques Poulin», Metka Zupancic (dir.), Mythes dans la littérature contemporaine d'expression française, actes du colloque tenu à l'Université d'Ottawa du 24 au 26 mars 1994, Ottawa, Le Nordir, 1994, p. 76-84.

+ MORENCY, Jean, «L'errance dans le roman québécois», Québec français, n 97, printemps 1995, p. 81-84.

+ MORENCY, Jean, "25 ans de présence américaine dans le roman québécois», Études canadiennes/Canadian Studies, n 52, 2002, p. 197-208.

+ MORENCY, Jean, "L'américanité et l'américanisation du roman québécois. Réflexions conceptuelles et perspectives littéraires», Globe. Revue internationale d'études québécoises, vol. VII, n 2, 2004, p. 31-58.

+ MORENCY, Jean, «Les appartenances culturelles et littéraires de Poulin», Larry Steele, Sophie Beaulé et Joëlle Cauville (dir.), Appartenances dans la littérature francophone d'Amérique du Nord, actes du colloque tenu à Halifax les 18 et 19 octobre 2002, Ottawa, Le Nordir, 2005, p. 135-144.

+ MORENCY, Jean, «La figure de Gabrielle Roy chez Jacques Poulin et Michel Tremblay», Canadian Literature, n 192, printemps 2007, p. 97-109.

+ MORENCY, Jean, «Images de l'Amérindien dans le roman québécois depuis 1945 », Tangence, $n^{\circ} 85$, automne 2007, p. 83-98.

+ MORENCY, Jean, «Dérives spatiales et mouvances langagières. Les romanciers contemporains et l'Amérique canadienne-française», Francophonies d'Amérique, n 26, 2008, p. 27-39.

+ MORENCY, Jean, «Une histoire dont vous n'êtes pas le héros. Le vieux Chagrin de Jacques Poulin », Frances Fortier et Andrée Mercier (dir.), La transmission narrative. Modalités du pacte romanesque contemporain, Québec, Nota bene, coll. «Contemporanéités», 2011, p. 97-110.

+ MORENCY, Jean, «Entre américanité et francité: Les yeux bleus de Mistassini, de Jacques Poulin ", Interculturel francophonies, n 32, novembre-décembre 2017, p. 147-159.

+ NAUCHE, Bénédicte, «Marika ou la féerie poulinienne», Hélène Noirot et Anne Giaufret (dir.), Actes du premier colloque de l'Association des jeunes chercheurs européens en littérature québécoise (28 et 29 avril 1993), Paris, Centre de coopération interuniversitaire francoquébécoise, Université Paris 7, 1993, p. 113-117.

+ NAVARRO PARDIÑAS, Blanca, «La représentation de la lecture dans Volkswagen Blues de Jacques Poulin», François Dumont et Frances Fortier (dir.), Littérature québécoise: la recherche en émergence, actes du deuxième colloque interuniversitaire de l'Association des jeunes chercheur(e)s en littérature québécoise tenu les 13, 14 et 15 juin 1990 au Centre de recherche en littérature québécoise de l'Université Laval, Québec, Nuit blanche éditeur, coll. «Les Cahiers du Centre de recherche en littérature québécoise de l'Université Laval», 1991, p. 71-80.

+ NAVARRO PARDIÑAS, Blanca, «Phénoménologie de l'acte de lecture. L'exemple de Volkswagen Blues de Jacques Poulin», Tangence, $n^{\circ}$ 36, mai 1992, p. 52-62.

+ NAVARRO PARDIÑAS, Blanca, «L'Amérique lunatique. Représentation des États-Unis dans quatre romans québécois», Hélène Noirot et Anne Giaufret (dir.), Actes du premier colloque de l'Association des jeunes chercheurs européens en littérature québécoise (28 et 29 avril 1993), Paris, Centre de coopération interuniversitaire franco-québécoise, Université Paris 7, 1993, p. 31-38.

+ NAVARRO PARDIÑAS, Blanca, «Hemingway au Québec. Voyage dans l'intertexte romanesque de Jacques Poulin», River Review/Revue Rivière, n² 2, 1996, p. 43-57. 
+ NAVARRO PARDIÑAS, Blanca, «La dérive des mots. Connotation et dénotation dans les romans de J. Poulin", River Review/Revue Rivière, Université du Maine à Fort Kent, 1999, s. p.

+ PAGLIUCA, Maria, "Poulin, Volkswagen Blues, une quête d'identité», Giovanni Dotoli, Il Canada del nuovo secolo. Gli archivi della memoria, actes du Convegno internazionale tenu à Monopoli du 30 mai au 3 juin 2001, Fasano, Schena editore, 2002, p. 363-373.

+ PARÉ, François, «Histoires de revenances», Voix et Images, vol. XXXIII, n 1, automne 2007, p. 147-151.

+ PARRIS, David L., "Cats in the Literature of Quebec. N'est pas chat qui le veut», British Journal of Canadian Studies, vol. III, n 2, 1988, p. 259-266.

+ PATERSON, Janet M., «Le vieux Chagrin, une histoire de chats? Ou comment déconstruire le postmoderne», Louise Milot et Jaap Lintvelt (dir.), Le roman québécois depuis 1960. Méthodes et analyses, Québec, Presses de l'Université Laval/Centre de recherche en littérature québécoise, 1992, p. 181-193.

+ PATERSON, Janet M., «Le postmodernisme québécois. Tendances actuelles», Études littéraires, vol. XXVII, no 1, été 1994, p. 77-88.

+ PATERSON, Janet M., «Ni l'un ni l'autre. L'ambivalence du discours de l'hétérogène dans Volkswagen Blues», University of Toronto Quaterly, vol. LXIII, n 4, été 1994, p. 605-613.

+ PEDRI, Nancy, «Cartographic explorations of self in Michael Ondaatje's Running in the family and Poulin's Volkswagen Blues », International Journal of Canadian Studies/Revue internationale d'études canadiennes, $\mathrm{n}^{\circ}$ 38, 2008, p. 41-60.

+ PICCIONE, Marie-Lyne, "Au rendez-vous des doublures: Volkswagen Blues de Jacques Poulin», Annales du Centre de recherches sur l'Amérique anglophone, vol. XXI, 1996, p. 121-128.

+ PICCIONE, Marie-Lyne, «Les références culturelles françaises dans Volkswagen Blues de Jacques Poulin », Jean-Pierre Bardet et René Durocher (dir.), Français et Québécois : le regard de l'autre (Paris, les 7, 8 et 9 octobre 1999), Paris, Centre de coopération interuniversitaire francoquébécoise, 1999, p. 321-325.

+ PICCIONE, Marie-Lyne, «Contournement et détournement de violence dans Chat sauvage de Poulin ", Sandrine Bazile et Gérard Peylet (dir.), Violence et écriture, violence de l'affect, voix de l'écriture, Pessac, Presses de l'Université de Bordeaux, coll. «Eidolon», 2008, p. 255-260.

+ PLOCHER, Hanspeter, «La littérature moderne du Québec. Son orientation vers le fantastique», Ingo Kolboom, Maria Lieber et Edward Reichel (dir.), Le Québec: société et cultures. Enjeux d'une francophonie lointaine, Dresden, Dresden University Press, 1998, p. 129-137.

+ PURDY, Anthony, «Memory Maps: Mnemotopic Motifs in Creates, Poulin, and Robin», Essays on Canadian Writing, no 80, automne 2003, p. 261-282.

+ RICARD, François, «Jacques Poulin: de la douceur à la mort», Liberté, vol. XVI, nºs 5-6, 1974, p. 97-105.

+ RICARD, François, «Jacques Poulin: Charlie Brown dans la Bible», Liberté, vol. XX, n 3, 1978, p. 85-88.

+ RICHARD, Chantal, «Analyse des alternances de langues par le logiciel Sphinx. Le cas du roman Volkswagen Blues de Jacques Poulin», Sylvia Kasparian et James De Finney (dir.), L'analyse des données textuelles: de l'enquête aux corpus littéraires, Moncton, Université de Moncton, 2004, p. $45-58$.

+ RICHARD, Chantal, «Vers une typologie de l'hétérolinguisme littéraire à la fin du xxe siècle en Amérique francophone», Jean Morency, Hélène Destrempes, Denise Merkle et Martin Pâquet 
(dir.), Des cultures en contact. Visions de l'Amérique du Nord francophone, Québec, Nota bene, coll. «Terre américaine», 2005, p. 249-263.

+ RIENDEAU, Pascal, «De la nostalgie d'un monde possible à la possible fin du monde», Voix et Images, vol. XXXV, $\mathrm{n}^{\circ}$ 1, automne 2009, p. 120-125.

+ RIENDEAU, Pascal, «Retours sur l'identité et la liberté», Voix et Images, vol. XL, nº 3, printemps-été 2015, p. 133-138.

+ ROBERTS, Paula, «Volkswagen Blues: redécouvrir l'histoire américaine», Chimères, n² 21, mars 1994, p. 49-65.

+ ROBERTS, Paula, «L'androgyne poulinien(ne): créature des marges?», La marge, actes du sixième colloque de la Société des études supérieures, Études françaises, Université de Toronto, 20-21 mai 1995, Toronto, SESDEF, 1997, p. 44-51.

+ ROCHON, François, «Nourriture, douceur et désespoir dans Les grandes marées de Jacques Poulin», Voix et Images, vol. XXXIV, n 3, printemps-été 2009, p. 81-97.

+ ROSENSTREICH, Susan L., "God the Father or Mother Earth? Nouvelle France in Two Quebec Novels of the 1980s", L'esprit créateur, vol. XLVIII, nº 1, printemps 2008, p. 120-130.

+ ROUSSEAU, Guildo, «La descente du continent», Revue internationale d'études canadiennes, $n^{\circ}$ 15, printemps 1997, p. 67-84.

+ SAINT-MARTIN, Lori, «L'androgynie, la peur de l'autre et les impasses de l'amour. La tournée d'automne de Jacques Poulin", Voix et Images, vol. XXIV, n³, printemps 1999, p. 541-557.

+ SAINT-MARTIN, Lori, "Romans d'homme, voix de femme. "Marie Auger", Gilles Archambault, Jacques Poulin et Maxime Mongeon», Voix et Images, vol. XXXII, n 2, hiver 2007, p. 31-47.

+ SAMOYAULT, Tiphaine, "Référence et post-modernité: Jacques Poulin», Littérature, n 113 , mars 1999, p. 115-123.

+ SAMZUN, Marie-Béatrice, «Le corps poulinien à l'épreuve du temps ou quand le miroir se brise», Daniel Marcheix et Nathalie Watteyne (dir.), L'écriture du corps dans la littérature québécoise depuis 1980, Limoges, Presses universitaires de Limoges, coll. «Espaces humains», 2007, p. 165-175.

+ SANAKER, John Kristian, « Jacques Poulin: l'art de la banalité, ou comment dire son amour mine de rien. Quelques remarques sur le dialogue de Volkswagen Blues et de La tournée d'automne», Vives lettres, n 10, 2000, p. 113-131.

+ SANAKER, John Kristian, "Jacques Poulin, romancier historien - sans en avoir l'air», Beïda Chikhi et Marc Quaghebeur (dir.), Les écrivains francophones interprètes de l'Histoire. Entre filiation et dissidence, Bruxelles, Archives et musée de la littérature/P.I.E. Peter Lang, coll. «Documents pour l'histoire des francophonies. Théorie», 2006, p. 207-215.

+ SANAKER, John Kristian, "Les yeux bleus de Mistassini de Jacques Poulin», Gilles Dupuis et Klaus-Dieter Ertler (dir.), À la carte. Le roman québécois (2000-2005), Frankfurt am Main, Peter Lang, 2007, p. 313-335.

+ SANAKER, John Kristian, «Volkswagen Blues de Jacques Poulin: des histoires pour écrire l'Histoire, ou des histoires pour former les personnages?», Lucie Hotte (dir.), (Se) Raconter des histoires. Histoire et histoires dans les littératures francophones du Canada, Sudbury, Prise de parole, coll. «Agora», 2010, p. 619-635.

+ SAPP, Robert, «Linguistic Vagabondage: The Driving Force in Jacques Poulin's Volkswagen Blues», Romance Notes, vol. XLVIII, n³ 3, 2008, p. 345-353.

+ SHODA-FUJIZANE, Yasuko, "Voyage et écriture chez Catherine Safonoff et Jacques Poulin", Martin Doré et Doris Jakubec (dir.), Deux littératures francophones en dialogue. Du Québec et 
de la Suisse romande, Québec, Presses de l'Université Laval, coll. «Études littéraires», 2004, p. 61-71.

+ SING, Pamela V., «McLuhan et Poulin: le village, côté cool», Benoît Melançon et Pierre Popovic (dir.), Miscellanées en l'honneur de Gilles Marcotte, Montréal, Fides, 1995, p. 267-279.

+ SIROIS, Antoine, "Espaces intimes et androgynie chez Jacques Poulin», Jaap Lintvelt et François Paré (dir.), Frontières flottantes. Lieu et espace dans les cultures francophones du Canada/Shifting Boundaries. Place and Space in the Francophones Cultures of Canada, Amsterdam/New York, Rodopi, coll. «Faux titre», 2001, p. 181-189.

+ SOCKEN, Paul G., "Water Imagery in the Novels of Jacques Poulin», Studies in Canadian Literature/Études en littérature canadienne, vol. XVIII, nº 2, 1993, p. 156-167.

+ SOCKEN, Paul G., «Le mythe au Québec à l'époque moderne», Metka Zupančič (dir.), Mythes dans la littérature contemporaine d'expression française, actes du colloque tenu à l'Université d'Ottawa du 24 au 26 mars 1994, Ottawa, Le Nordir, 1994, p. 51-56.

+ SOCKEN, Paul G., «Jacques Poulin: héritier spirituel de Gabrielle Roy », André Fauchon (dir.), Colloque international «Gabrielle Roy», actes du colloque soulignant le cinquantième anniversaire de Bonheur d'occasion (27 au 30 septembre 1995), Saint-Boniface, Presses universitaires de Saint-Boniface, 1996, p. 593-603.

+ SOCKEN, Paul G., «Jacques Poulin's Le cœur de la baleine bleue: A Rewriting of Gabrielle Roy's Alexandre Chenevert?», IJFS, n² 2, 1999, p. 106-111.

+ SORON, Anthony, «Volkswagen Blues de Poulin. La reconquête de la frontière imaginaire», Eidôlon, n 67, 2004, p. 371-380.

+ STRATFORD, Philip, "No Clear Strait of Anian: Comparing Jacques Poulin and Matt Cohen», Arnold E. Davidson (dir.), Studies on Canadian Literature: Introductory and Critical Essays, New York, Modern Language Association of America, 1990, p. 296-308.

+ THIBEAULT, Jimmy, «L'invention de la Franco-Amérique. La relecture de l'Histoire en histoires chez Antonine Maillet et Jacques Poulin", Québec Studies, n 53, avril 2012, p. 9-27.

+ THOMAS, Yves, "La part des labels et des marchandises dans Les grandes marées», Voix et Images, vol. XV, $\mathrm{n}^{\circ} 1$, automne 1989, p. 43-50.

+ TREMBLAY, Roseline, "Le vieux Chagrin de Jacques Poulin: l'écrivain de l'entre-deux", Québec Studies, $\mathrm{n}^{\circ} 38$, octobre 2004, p. 37-46.

+ TREMBLAY, Victor-Laurent, «Masculinité et hockey dans le roman québécois», The French Review, vol. LXXVIII, n 6 , mai 2005, p. 1104-1116.

+ TRUJILLO GONZÁLEZ, Verónica Cristina, "Approche onomastique de Les grandes marées de Jacques Poulin", Çédille. Revista de Estudios Franceses, nº 6, avril 2010, p. 245-256.

+ VANDERVOORT, Edith Biegler, "Subverting the Masculine Image in Jacques Godbout's Salut Galarneau and Jacques Poulin's Volkswagen Blues», Edith Biegler Vandervoort (dir.), Masculinities in Twentieth- and Twenty-First Century French and Francophone Literature, Newcastle upon Tyne, Cambridge Scholars, 2011, p. 305-324.

+ VAN’T LAND, Hilligje, «La "Kérouacquisition" du territoire américain. Poulin, Godbout, et LaRue comme représentants du discours littéraire québécois des années 80 », Het Franse Boek, $n^{\circ}$ 66, 1996, p. 31-40.

+ VAUTIER, Marie, «Postmodern Myth, Post-European History, and the Figure of the Amerindian. François Barcelo, George Bowering and Jacques Poulin», Canadian Literature, no 141, été 1994, p. 15-33. 
+ VAUTIER, Marie, «Canadian Fiction Meets History and Historiography: Jacques Poulin, Daphne Marlatt, and Wayson Choy", Colby Quarterly, vol. XXXV, n 1, mars 1999, p. 18-34.

+ VOICULESCU, Liliana, «La voiture: espace identitaire chez Jacques Poulin», Annales Universitatis Apulensis. Series Philologica, vol. IX, n² 2, 2008, p. 25-30.

+ WALKER, David, «The Role of the Incipits in Poulin's Le vieux Chagrin», LittéRéalité, vol. VI, $n^{\circ} 1$, printemps-été 1994, p. 97-107.

+ WEISMAN, Adam Paul, «Postcolonialism in North America: Imaginative Colonization in Henry David Thoreau's A Yankee in Canada and Jacques Poulin's Volkswagen Blues », Massachusetts Review: A Quarterly of Literature, the Arts and Public Affairs, vol. XXXVI, n³, automne 1995, p. $477-500$.

+ WEISS, Jonathan M., «Une lecture américaine de Volkswagen Blues», Études françaises, vol. XXI, n 3, hiver 1985, p. 89-96.

+ WEISS, Jonathan, «Jacques Poulin, lecteur de Hemingway », Études françaises, vol. XXIX, nº 1 , printemps 1993, p. 11-23.

+ WINTERBURN, Mike, " "Américanité" in two Recent Quebec Novels», British Journal of Canadian Studies, vol. III, nº 2, 1988, p. 277-284.

+ YELLĖS, Mourad, «Volkswagen Blues de Jacques Poulin», Bouba Mohammedi Tabti (coordonnatrice), Littérature canadienne. Impressions de lecture, Blida (Algérie), Éditions du Tell, 2005, p. 97-102.

+ YOTOVA, Rennie, «Jacques Poulin, Volkswagen Blues (1984)», Pierre Morel (dir.), Parcours québécois. Introduction à la littérature du Québec, Kichinez, Cartier, 2007, p. 144-155.

\section{Portraits}

+ BEAULIEU, Nicole, «L'écrivain dans l'ombre», L'Actualité, vol. X, n 4, avril 1985, p. 73-77.

+ BOURQUE, Paul-André, "Actualité de Jacques Poulin», Lettres québécoises, n 83, automne 1996, p. 8-10.

+ CARRIER, Roch, «Jacques Poulin: Toronto-Montréal», Nord, n² 2, hiver 1972, p. 73.

+ CRÉPEAU, Jean-François, «Un art ou un style nommé Poulin», Lettres québécoises, n 123, automne 2006, p. 25.

+ HAMEL, Réginald, John HARE et Paul WYCZYNSKI, «Poulin, Jacques», Dictionnaire pratique des auteurs québécois, Montréal, Fides, 1976, p. 568-569.

+ HAMEL, Réginald, John HARE et Paul WYCZYNSKI, «Poulin, Jacques», Dictionnaire des auteurs de langue française en Amérique du Nord, Montréal, Fides, 1989, p. 1115.

+ JANELLE, Claude, Les Éditions du Jour: une génération d'écrivains, préface d'André Major, Montréal, Hurtubise HMH, coll. «Cahiers du Québec. Littérature», 1983, s. p.

+ MAILHOT, Laurent et Sheila FISCHMAN, «Jacques Poulin», Eugene Benson (dir.), Oxford Companion to Canadian Literature, Oxford, Oxford University Press, 1983, p. 675-676.

+ SÉVIGNY, Marie-Ėve, «Jacques Poulin - "Réchauffer le cœur de quelqu'un”», Entre les lignes, vol. VIII, n 2, hiver 2012, p. 26-27.

\section{Comptes rendus et articles de presse (bibliographie non exhaustive)}

\section{a) Mon cheval pour un royaume}

+ BILODEAU, Line, "Mon cheval pour un royaume», Maurice Lemire (dir.), Dictionnaire des œuvres littéraires du Québec, t. IV : 1960-1969, Montréal, Fides, 1984, p. 585-587. 
+ DESROSIERS, Jacques, «Hertel pour un cheval», L'Écho du Nord, 17 janvier 1968, p. 38 et p. 55.

+ ÉTHIER-BLAIS, Jean, «Mon cheval pour un royaume de Jacques Poulin», Le Devoir, 30 juin 1967, p. 15.

+ GAY, Paul, «Le cheval de Jacques Poulin», La Presse, 30 juin 1967, p. 16.

+ LEROUX, Odette, "Mon cheval pour un royaume de Jacques Poulin», Livres et auteurs québécois, 1967, p. 58.

+ MAJOR, André, "Mon cheval pour un royaume», Le Devoir, 8 juin 1967, p. 6.

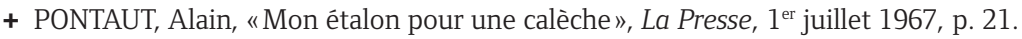

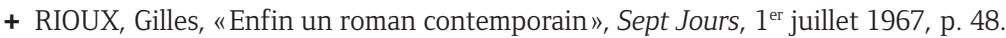

+ SAINT-ONGE, Paule, «Mon cheval pour un royaume», Châtelaine, vol. VIII, n 9, septembre 1967, p. 28.

+ VALIQUETTE, Bernard, «Mon cheval pour un royaume», Échos Vedettes, 19 août 1967, p. 21.

\section{b ) Jimm}

+ BEAULIEU, Victor-Lévy, «De la fiction à l'affliction», Digeste éclair, vol. VI, n 4, avril 1969, p. 17.

+ BONIN, Raymond, «Jimmy de Jacques Poulin», Les Cahiers François-Xavier-Garneau, nº 2 1970, p. 76-78. Article reproduit dans Le Lien, janvier 1970, p. 10.

+ BOSCO, Monique, «Un menteur infiniment poétique», Maclean's, mai 1969, p. 70.

+ BOURQUE, Paul-André, «Jacques Poulin ou l'art de communiquer l'incommunicabilité», Québec français, n 34, mai 1979, p. 38-39.

+ COSSETTE, Gilles, «Jimmy», Livres et auteurs québécois, 1969, p. 12-13.

+ ÉTHIER-BLAIS, Jean et Gilles DORION, «Jimmy», Maurice Lemire (dir.), Dictionnaire des œuvres littéraires du Québec, t. IV : 1960-1969, Montréal, Fides, 1984, p. 469-471.

+ GAY, Paul, «Jimmy», Le Droit, 19 juillet 1969, p. 7.

+ MAJOR, André, «Jimmy», Le Devoir, 15 mars 1969, p. 15.

+ MARCOTTE, Gilles, «Comptes rendus. Jacques Poulin. Jimmy», Études françaises, vol. V, nº 2, mai 1969, p. 236-237.

+ MARCOTTE, Gilles, "Poulin chez les hommes (Jimmy)», Les bonnes rencontres. Chroniques littéraires, Montréal, Hurtubise HMH, coll. «Reconnaissances», 1971, p. 205-207.

+ MARTEL, Réginald, «C'est d'enfance qu'il s'agit», La Presse, 8 mars 1969, p. 23.

+ ROUX, Paul, «Jimmy: un grand roman sur l'enfance», Le Soleil, 15 mars 1969, p. 32.

+ THÉBERGE, Jean-Yves, «L'excellent Jimmy», Le Canada français, 28 mai 1969, p. 44.

\section{c) Le cœur de la baleine bleue}

+ ANONYME, «Le Québec de Jacques Poulin», Entre les lignes, vol. IV, n 4, été 2008, p. 20-21.

+ ANONYME, "Le cœur de la baleine bleue», Le livre canadien, vol. II, n 85, 1971, s. p.

+ ANONYME, «Le cœur de la baleine bleue», L'Église canadienne, décembre 1972, p. 308.

+ DEMERS, Jeanne, «Le cœur de la baleine bleue de Jacques Poulin», Livres et auteurs québécois, 1970, p. 46-47.

+ ÉTHIER-BLAIS, Jean, «Le cœur (emprunté) de la baleine bleue», Le Devoir, 23 janvier 1971, p. 11.

+ GARNEAU, Jacques, "Le cœur de la baleine bleue», Maurice Lemire (dir.), Dictionnaire des œuvres littéraires du Québec, t. V: 1970-1975, Montréal, Fides, 1987, p. 161-162.

+ HANDFIELD, Micheline, "Le cœur de la baleine bleue: l'histoire d'une enfance inaccessible», Québec presse, 17 janvier 1971, p. B18. 
+ LABERGE, Pierre, «Le rejet. À propos du Cœur de la baleine bleue», L'Action, 20 février 1971, p. 19.

+ POULIN, Gabrielle, "Le cœur de la baleine bleue de Jacques Poulin, un roman piégé», Relations, mai 1972, p. 154-155. Article reproduit dans Romans du pays, 1968-1979, avec des textes de René Dionne, Montréal, Bellarmin, 1980, p. 263-270.

+ THÉBERGE, Jean-Yves, «Une histoire de cœur, mais du cœur d'une autre», Le Canada français, 17 février 1971, p. 26.

\section{d) Faites de beaux rêves}

+ ANONYME, "Faites de beaux rêves», Le livre canadien, vol. V, n 320, 1974, s. p.

+ BOURNEUF, Roland, «Jacques Poulin, Faites de beaux rêves", Livres et auteurs québécois, 1974, p. 51-53.

+ GALLAYS, François, "Faites de beaux rêves", Maurice Lemire (dir.), Dictionnaire des œuvres littéraires du Québec, t. V: 1970-1975, Montréal, Fides, 1987, p. 332-333.

+ MARCOTTE, Gilles, «Lisez Jacques Poulin, Faites de beaux rêves», Le Devoir, 12 mai 1979, p. 23.

+ PELLETIER, Jacques, «Faites de beaux rêves», Livres et auteurs québécois, 1974, p. 17.

\section{e) Les grandes marées}

+ CHAPUT, Sylvie, "Les grandes marées», La revue Pantoute, nº 2, juin-juillet-août 1980, p. 24.

+ DORION, Gilles, "Les grandes marées», Québec français, n 31, octobre 1978, p. 6.

+ DUQUETTE, Jean-Pierre, "Les grandes marées», Livres et auteurs québécois, 1978, p. 19.

+ GODBOUT, Jacques, «Les grandes marées», L'Actualité, vol. III, nº 8, août 1978, p. 55.

+ MONTESSUIT, Carmen, «Les grandes marées», Journal de Montréal, 29 novembre 1981, p. 62.

+ NOLIN, Jacques, «Les grandes marées», Nos livres, vol. IX, n 250, juin-juillet 1978, s. p.

+ OUELLETTE, Gabriel-Pierre, "Jacques Poulin: Les grandes marées", Livres et auteurs québécois, 1978, p. 71-74.

+ OUELLETTE-MICHALSKA, Madeleine, «Les grandes marées», Châtelaine, vol. XIX, nº 8, août 1978, p. 16.

+ PAGÉ, Raymond, «Les grandes marées», The Chelsea Journal, vol. V, no 5, septembre-octobre 1979, p. 232-233.

+ POULIN, Gabrielle, «Les grandes marées de Jacques Poulin ou la Genèse... en bandes dessinées», Relations, nº 437, mai 1978, p. 154-156.

+ RENAUD, André, «Les grandes marées», Voix et Images, vol. V, nº 1, automne 1979, p. 193-195.

\section{f) The Jimmy Trilogy}

+ ANONYME, «The Jimmy Trilogy», Choice, vol. XVII, mai 1980, p. 395.

+ CARRIER, Roch, «Introduction», Jacques Poulin, The Jimmy Trilogy, traduction anglaise de Sheila Fischman, Toronto, House of Anansi Press, 1979, p. 6-7.

+ DAVIES, G., «The Jimmy Trilogy: Book Review», The Fiddlehead, nº 129, printemps 1981, p. 128-129.

+ GRADY, Wayne, «The Jimmy Trilogy: Accent on the Soul», Books in Canada, vol. IX, $\mathrm{n}^{\circ}$ 1, janvier 1980, p. 14-15.

+ HUNT, Douglas, «The Jimmy Trilogy», Quill \& Quire, vol. LXVI, nº 4, avril 1980, p. 34.

+ MERIVALE, Patricia, «The Jimmy Trilogy», Canadian Literature, nº 88, printemps 1981, p. 128-129. 
+ MEZEI, Kathy, «The Jimmy Trilogy», Queen's Quarterly, vol. LXXXVII, n 3, automne 1980, p. 518-519.

+ PHILPOTTS, Jane, «Quiet Reading of Jacques Poulin's Trilogy Leaves Pleasant Taste», Calgary Herald, 29 décembre 1979, p. C10.

+ RASPORICH, Beverly, «The Jimmy Trilogy», Canadian Book Review Annual, 1979, p. 103.

\section{g) Volkswagen Blues}

+ BEKKAT, Amina Azza, «Volkswagen Blues de Jacques Poulin», Bouba Mohammedi Tabti (coordonnatrice), Littérature canadienne. Impressions de lecture, Blida (Algérie), Éditions du Tell, 2005, p. 93-96.

+ DE BILLY, Hélène, «Une Amérique panoramique sur la pointe des pieds», Le Devoir, 19 mai 1984, p. 25 et p. 33.

+ CHASSAY, Jean-François, «Un écrivain américain», Spirale, septembre 1984, p. 8.

+ DÉCARIE, Nicole, «Volkswagen Blues», Mœbius, n 22, été 1984, p. 100-101.

+ DORION, Gilles, "Volkswagen Blues», Québec français, n 55, octobre 1984, p. 8.

+ GAUDET, Gérald, «Adolescente Amérique», Le Sabord, n 4, automne 1984, p. 6.

+ GILBERT, Bernard, «Volkswagen Blues», Inter, n 25, automne 1984, p. 52.

+ LAURIN, Michel, «Le livre du mois: Volkswagen Blues de Jacques Poulin», Le Progrès, 30 juillet 1984, p. 15.

+ LAURIN, Michel, «Volkswagen Blues», Nos livres, vol. XV, n5 5774-5823, juin-juillet 1984, s. p.

+ MARCOTTE, Gilles, «L'ambitieuse Volkswagen», L'Actualité, vol. IX, n 8, août 1984, p. 79.

+ MARTEL, Réginald, "L'Amérique entre rêve et violence», La Presse, 12 mai 1984, p. D3.

+ MELANÇON, Benoît, "Roman américain, Jacques Poulin: Volkswagen Blues», Canadian Literature, no 103, hiver 1984, p. 111-113.

+ MILOT, Louise, "Quand les "beaux rêves" tournent au "blues". Le Volkswagen de Jacques Poulin», Lettres québécoises, n 35, automne 1984, p. 15-17.

+ RUDEL-TESSIER, Danièle, "Volkswagen Blues», Châtelaine, vol. XXV, n 9, septembre 1984, p. 43.

+ TASCHEREAU, Yves, «Volkswagen Blues», Livre d'ici, vol. IX, n 9, mai 1984, p. 23.

+ TREMBLAY, Régis, «Poulin: la fraternité est la nouvelle frontière», Le Soleil, 7 juillet 1984, p. D1.

+ TREMBLAY, Régis, «Déshabiller la phrase de ses adjectifs», Le Soleil, 7 juillet 1984, p. D2.

+ TREMBLAY, Yolaine, «Volkswagen Blues par Jacques Poulin», Livre d'ici, vol. IX, n 9, mai 1984, p. 23.

+ VASSEUR, François et Michelle ROY, «Voyage à travers l'Amérique», Nuit blanche, nº 14, juinaoût 1984, p. 50-52.

+ YELLĖS, Mourad, «Volkswagen Blues de Poulin», Bouba Mohammedi Tabti (coordonnatrice), Littérature canadienne. Impressions de lecture, Blida (Algérie), Éditions du Tell, 2005, p. 97-102.

\section{h) Spring Tides}

+ ABLEY, Mark, «Trouble in Paradise: Review of Spring Tides», Maclean's, $1^{\mathrm{er}}$ septembre 1986, p. 56.

+ ADACHI, Ken, «Quebec Fiction: A Lively Book and a Bleak One: Review of Spring Tides and Standing Flight», Toronto Star, 10 août 1986, p. A19.

+ ANDREWS, Audrey, «Tender Fable Reveals Man's Loneliness: Review of Spring Tides», Calgary Herald, 21 septembre 1986, p. C8. 
+ FRENCH, William, "Quebec Changes: Reviews of Spring Tides and The Legacy», The Globe and Mail, 26 juillet 1986, p. D15.

+ NACHTSHEIM, M. H., «Spring Tides», Choice, vol. XXIV, février 1987, p. 889.

+ QUIGLEY, Theresa, "A crowded Eden", The Fiddlehead, n 153, automne 1987, p. 102-103.

+ RAFELMAN, Rachel, «Spring Tides», Quill \& Quire, vol. LII, octobre 1986, p. 46.

+ SPETTIGUE, D. O., «Spring Tides; Jacques Poulin; Book Review», Queen's Quarterly, vol. XCIV, no 2, été 1987, p. 366-375.

+ URQUART, J., «Spring Tides by Jacques Poulin: Book Review», Canadian Literature, nº 116, printemps 1988, p. 129-131.

+ WILLIAMSON, David, "Gentle Fable of a Lost Eden: Review of Spring Tides», Winnipeg Free Press, 9 août 1986, p. 50.

\section{i) Volkswagen Blues (version anglaise)}

+ ACKERMAN, Marianne, "The French Connection: Review of Volkswagen Blues", Maclean's, 16 mai 1988, p. 58.

+ ADACHI, Ken, «Poulin's Road Trip is a Kerouac-Like Odyssey», Sunday Star, 8 mai 1988, p. A22.

+ CASSIDY, Jim, "Great Characters in Need of Further Study», Vancouver Sun, 16 avril 1988, p. D4.

+ FRENCH, William, "Quebec Novel Takes a Journey in a Dream Machine», The Globe and Mail, 14 avril 1988, p. A20.

+ GOLDIE, Terry, «Transcontinental», Books in Canada, vol. XVIII, nº 6, août-septembre 1988, p. 217.

+ JARMAN, Marc Anthony, «Volkswagen Blues; Jacques Poulin», Malahat Review, n 83, 1988, p. 187-188.

+ JARMAN, Marc Anthony, «This Slow Volkswagen Needs Turbocharging», Calgary Herald, 28 août 1988, p. F5.

+ LEDGER, Brent, «Poulin's Witty Trek: Quebec's Reach for an American Home», Quill \& Quire, vol. LIV, nº 6, juin 1988, p. 26.

+ SIMON, Sherry, "Quebec Writer, Metis Girl, Go to Look for America», The Montreal Gazette, 21 mai 1988, p. L12.

+ WILLIAMSON, David, «Quebec Novelist Offers Lesson in Joyous, Gentle Writing», Winnipeg Free Press, 30 avril 1988, p. 7A.

\section{j) Le vieux Chagrin}

+ BEAUDOIN, Réjean, «Le vin de la tendresse», Liberté, vol. XXXII, n 2, avril 1990, p. 94-104.

+ BERERHI, Afifa, «Le vieux Chagrin de Poulin», Bouba Mohammedi Tabti (coordonnatrice), Littérature canadienne. Impressions de lecture, Blida (Algérie), Éditions du Tell, 2005, p. 103-109.

+ BOIVIN, Aurélien, "Le vieux Chagrin ou une leçon d'écriture», Québec français, nº 138, été 2005, p. 94-97.

+ DORION, Gilles, «Un immense besoin de tendresse», Québec français, nº 78, été 1990, p. 76.

+ LEVASSEUR, Jean, "Le vieux Chagrin», Revue francophone de Louisiane, vol. V, n² 2, hiver 1990, p. 104-106.

+ MARTEL, Réginald, «Jacques Poulin et Le vieux Chagrin. La nouvelle version du chefd'œuvre», La Presse, 18 novembre 1989, s. p. 
+ MARTEL, Réginald, «Sur le sentier du Vieux Chagrin», La Presse, 24 mai 1992, p. C3.

+ MIRAGLIA, Anne Marie, «Le vieux Chagrin de Jacques Poulin», Voix et Images, vol. XVI, nº 1 , automne 1990, p. 170-172.

\section{k) La tournée d'automne}

+ ALLARD, Jacques, "Dieu soit remercié pour les romans de Poulin», Le Devoir, 13 novembre 1993, p. D5.

+ CAYOUETTE, Pierre, «Pas la peine de compter, Jack!», Le Devoir, 6 novembre 1993, p. D9.

+ CHASSAY, Jean-François, «Topographies américaines», Voix et Images, vol. XIX, n² 2, hiver 1994, p. 416-420.

+ LEVASSEUR, Jean, «La tournée d'automne», Revue francophone, vol. VIII, n² 2, 1993, p. 118-120.

+ LÉVESQUE, Robert, «Jacques Poulin. Un ermite à Paris», Le Devoir, 20 novembre 1993, p. D1.

+ MARCOTTE, Gilles, «Une aventure risquée et... réussie», L'Actualité, vol. XIX, nº 1, janvier 1994, p. 70.

+ MARTEL, Réginald, «Une embardée du cœur sauve la vie d'un chauffeur de bibliobus», La Presse, 14 novembre 1993, p. B7.

+ MICHAUD, Ginette, «Compte rendu du livre La tournée d'automne de J. Poulin», Spirale, nº 130, février 1994, p. 3 et p. 6.

+ STEVEN, Daniell, «La tournée d'automne», World Literature Today, n 69, 1995, p. 93-94.

+ VOISARD, Anne-Marie, «Même si vous n'aimez pas les chats, vous aimerez La tournée d'automne», Le Soleil, 6 décembre 1993, p. B7.

\section{1) Chat sauvage}

+ ANONYME, "Chat sauvage à suivre dans le Vieux-Québec», Le Progrès, 12 avril 1998, p. B6.

+ BIRON, Michel, «Des héros très discrets», Voix et Images, vol. XXIV, n 1, automne 1998, p. 197-202.

+ CHARTRAND, Robert, "L'exil comme un chez-soi», Le Devoir, 21 mars 1998, p. D3.

+ LACHANCE, Lise, «Jacques Poulin. Nostalgie parisienne», Le Soleil, 15 mars 1998, p. B10.

+ LEVASSEUR, Jean, «Poulin, Chat sauvage», The French Review, nº 73, 1999-2000, p. 595-596.

+ MARCOTTE, Gilles, «Le Chat sauvage», L'Actualité, vol. XXIII, n 10, juin 1998, p. 97.

+ NAVARRO PARDIÑAS, Blanca, "Chat sauvage de Jacques Poulin», Francophonies d'Amérique, n० 9, 1999, s. p.

+ NAVARRO PARDIÑAS, Blanca, "Poulin, Chat sauvage», Études. Revue de culture contemporaine, vol. XIV, n 1, printemps 1999, p. 227-232.

\section{m) Les yeux bleus de Mistassini}

+ BIRON, Michel, «La maladie d'Eisenhower», Le Devoir, 7 décembre 2002, p. F3.

+ CRÉPEAU, Jean-François, «La maladie des grands esprits et des cœurs fous», Lettres québécoises, nº 110, été 2003, p. 19-20.

+ DEITZ, Ritt, «Les yeux bleus de Mistassini», The French Review, nº 78, 2004-2005, p. 204-206.

+ GAUDREAU, Hélène, "Poulin, Les yeux bleus de Mistassini», Nuit blanche, no 91, été 2003, p. 17.

+ GIGUĖRE, Suzanne, «Le style, c'est l'âme», La Presse, 24 novembre 2002, p. F2.

+ LEVASSEUR, Jean, "Les yeux bleus de Mistassini», Nouvelles Études francophones, vol. XIX, $\mathrm{n}^{\circ} 2$, automne 2004, p. 272-274. 
+ MARCOTTE, Gilles, «L'immersion totale», L'Actualité, $1^{\text {er }}$ février 2003, p. 75.

+ MONTPETIT, Caroline, «Livres. Retour à Québec: Jacques Poulin», Le Devoir, 26 octobre 2002, s. p.

+ VIGNEAULT, Benny, «Orfèvre de l'écriture», Le Soleil, 27 octobre 2002, p. B3.

\section{n) La traduction est une histoire d'amour}

+ CASEVITZ, Michel, «Une histoire d'amour... », Conjonctures, n 43, hiver 2007, p. 201-203.

+ CRÉPEAU, Jean-François, «Un art ou un style nommé Poulin», Lettres québécoises, n 123, automne 2006, p. 25.

+ DESMEULES, Christian, «Jacques Poulin et la petite musique des mots», Le Devoir, section «Lire», 25 mars 2006, en ligne: https://www.ledevoir.com/lire/105124/ jacques-poulin-et-la-petite-musique-des-mots.

+ DESMEULES, Sabin, «Un roman d'amour», L'Acadie Nouvelle, 5 août 2006, p. ACCENT 2.

+ FESSOU, Didier, «L'entrevue est dans le livre», Le Soleil, 19 mars 2006, p. C1.

+ FORTIN, Marie-Claude, «Le cœur découvert», La Presse, 26 mars 2006, p. ARTS SPECTACLES 13.

+ GIGUÈRE, Suzanne, «Les mots doux», Le Devoir, 8 avril 2006, p. F3.

+ LESSARD, Valérie, "La petite musique des mots de Poulin», Le Droit, 18 mars 2006, p. A14.

+ LEVASSEUR, Jean, «Poulin, La traduction est une histoire d'amour», Nouvelles Études francophones, vol. XXII, $\mathrm{n}^{\circ}$ 1, printemps 2007, p. 224.

+ MARCOTTE, Gilles, «Deux plumes de la Vieille Capitale», L'Actualité, vol. XXXI, nº 12, août 2006, p. 73.

+ MIRAGLIA, Anne Marie, «Poulin, La traduction est une histoire d'amour», Canadian Literature/ Littérature canadienne. A Quarterly of Criticism and Review, n 191, hiver 2006, p. 191.

\section{o) L'anglais n'est pas une langue magique}

+ CORRIVEAU, Hugues, «Jacques Poulin, Jean Barbe, Anne Legault», Lettres québécoises, nº 134, été 2009, p. 22.

+ DESMEULES, Christian, «Littérature québécoise. Jacques Poulin, le roman et l'art du rapaillage», Le Devoir, 21 mars 2009, p. F3.

+ DROUIN, Serge, "Rédiger la réalité», Journal de Montréal, 18 avril 2009, p. W88.

+ FESSOU, Didier, «Comme un vieux copain...», Le Soleil, 22 mars 2009, p. 41.

+ LACROIX, Isabelle, "La poésie au quotidien», L'Acadie Nouvelle, 31 juillet 2010, p. SA2.

+ LAURIN, Danielle, «Magie de l'écriture», Le Devoir, 21 mars 2009, p. F3.

+ LAURIN, Danielle, «Mes meilleurs romans québécois des derniers mois», Le Devoir, 6 juin 2009, p. F3.

+ LEMERY, Marthe, «Un faux polar mais un vrai délice», Le Droit, 4 avril 2009, p. A14.

+ LEVASSEUR, Jean, «Poulin, L'anglais n'est pas une langue magique», Nouvelles Études francophones, vol. XXIV, nº 2, automne 2009, p. 209-210.

+ LÉVESQUE, Robert, "Amours durables, lectures tenaces», La Presse, 22 mars 2009, p. LECTURES 3.

+ NORMAND, Anne, "Drogue douce», La Voix de l'Est, 4 avril 2009, p. 32.

+ PARÉ, Yvon, «Jacques Poulin offre un moment de bonheur», Le Progrès, 24 mai 2009, p. 42.

+ PLAMONDON, Jean-François, «Poulin, L'anglais n'est pas une langue magique», Studi francesi, vol. LIII, no 3, 2009, p. 684. 
+ VOISARD, Anne-Marie, «Jacques Poulin: la langue de chez nous», Le Soleil, 22 mars 2009, p. 46.

\section{p) L'homme de la Saskatchewan}

+ BORNAIS, Marie-France, "Hymne au hockey... en français», Journal de Montréal, 26 novembre 2011, p. W104.

+ BROCHU, André, «Jacques Poulin, France Daigle», Lettres québécoises, n 145, printemps 2012, p. 18.

+ CRÉPEAU, Jean-François, «Le Poulin nouveau», Le Canada français, 5 janvier 2012, p. C6.

+ DESMEULES, Christian, "Jacques Poulin, l'homme et ses doubles», Le Devoir, 1" ${ }^{\text {er }}$ octobre 2011, F1.

+ LAURIN, Danielle, «L'exploit Jacques Poulin», Le Devoir, 8 octobre 2011, p. F3.

+ LESSARD, Valérie, "Réminiscences des luttes métisses», Le Droit, 15 octobre 2011, p. A13.

+ ROCHON, François, «Le nouveau blues de Jacques Poulin, L’homme de la Saskatchewan", Spirale, $\mathrm{n}^{\circ}$ 240, printemps 2012, p. 82-83.

+ TREMBLAY, Régis, «Le fantôme de l'écrivain", Le Soleil, $1^{\text {er }}$ octobre 2011, en ligne: https:// www.lesoleil.com/archives/jacques-poulin-lefantome-delecrivain-4624d293069cb080a18eb05a 43e389a6

\section{q) Un jukebox dans la tête}

+ BORNAIS, Marie-France, "Fragile histoire d'amour», Journal de Montréal, 8 février 2015, en ligne: www.journaldemontreal.com/2015/02/06/fragile-histoire-damour (page consultée le 8 novembre 2019).

+ BROCHU, André. «Entre l'amour et l'amitié. Robert Lalonde, Jacques Poulin, Guy Verville», Lettres québécoises, no 159, automne 2015, p. 24-25.

+ CRÉPEAU, Jean-François, «Jack Waterman nous visite», Le Canada français, 2 avril 2015, p. CAHC9.

+ DESMEULES, Christian, «Petite musique en boucle», Le Devoir, 7 février 2015, p. F3.

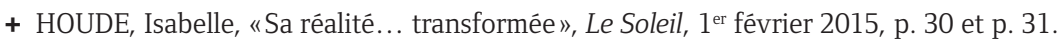

\section{E. Mémoires et $t h$ ès es}

+ AYOTTE, Marie-Josée, L'itinéraire spirituel du JE écrivain dans Le vieux Chagrin de Jacques Poulin: de l'enveloppement à la nudité, mémoire de maîtrise, Trois-Rivières, Université du Québec à Trois-Rivières, 1996, $129 \mathrm{f}$.

+ BERGERON, Francine, Le héros dans l'œuvre romanesque de Jacques Poulin, mémoire de maîtrise, Trois-Rivières, Université du Québec à Trois-Rivières, 1986, 182 f.

+ BERNIER, France, Du paratexte au texte: lire l'Amérique dans Volkswagen Blues de Jacques Poulin, mémoire de maîtrise, Québec, Université Laval, 1998, 105 f.

+ BERNOVSKY, Victor, La présence intertextuelle d'Ernest Hemingway dans trois romans de Jacques Poulin, mémoire de maîtrise, Vancouver, Université de la Colombie-Britannique, 2012, $129 \mathrm{f}$.

+ BISTODEAU, Linda, Sémantique littéraire de l'espace dans Volkswagen Blues de Jacques Poulin, mémoire de maîtrise, Trois-Rivières, Université du Québec à Trois-Rivières, 1989, 159 f.

+ BLANCHET-CHOUINARD, Julie, L'apprivoisement du pôle intérieur. Le rituel chez Jacques Poulin (comment le rituel sert-il la quête de l'anima et du bonheur), mémoire de maîtrise, Québec, Université Laval, 2002, 85 f. 
+ BONIN, Éric, Le signe Amérique chez Victor-Lévy Beaulieu et Jacques Poulin, mémoire de maîtrise, Montréal, Université de Montréal, 1993, 123 f.

+ BOUCHER, Hugo, L'emprunt du chemin de traverse entre Jimmy et Jim ou une lecture bachelardienne de Jimmy et du Vieux Chagrin de Jacques Poulin, mémoire de maîtrise, Sherbrooke, Université de Sherbrooke, 2011, 138 f.

+ BUJOLD, Marie-France, La mise en scène de l'interaction dans l'œuvre de Jacques Poulin, mémoire de maîtrise, Montréal, Université McGill, 2008, 119 f.

+ BUSSIÈRES GALLAGHER, Anne, Le traducteur fictif, personnage de la littérature québécoise, mémoire de maîtrise, Sherbrooke, Université de Sherbrooke, 2010, 115 f.

+ CAMPBELL, Catherine, Hearing the Silence: A Legacy of Post-Modernism, thèse de doctorat, Sherbrooke, Université de Sherbrooke, 2003, 212 f.

+ CAMPBELL, Elizabeth Margaret, Intertextualité: Hemingway chez Jacques Poulin, mémoire de maîtrise, Waterloo, Université de Waterloo, 1993, 124 f.

+ CHARLOT, Adeline, L'abolition de la dualité sexuelle chez Jacques Poulin: condition ou obstacle au bonheur? Étude de Volkswagen Blues et de La tournée d'automne, mémoire de maîtrise, Québec, Université Laval, 2007, 95 f.

+ CHARPIOT, Matthias, Les fonctions du voyage dans Volkswagen Blues de Jacques Poulin, mémoire de maîtrise, Paris, Université Paris IV-Sorbonne, 2003, $101 \mathrm{f}$.

+ CIOCHIN, Doina, Analyse et traduction en italien de La traduction est une histoire d'amour de Jacques Poulin, mémoire de maîtrise, Sherbrooke, Université de Sherbrooke, 188 f.

+ CONNOLLY, Carole, Manifestations du narrataire dans le roman québécois, thèse de doctorat, Ottawa, Université d'Ottawa, 1999, 252 f.

+ CRAINIC, Corina, Représentation de la bibliothèque chez Bessette et Poulin, mémoire de maîtrise, Montréal, Université McGill, 2004, 142 f.

+ CUBEDDU, Stefania, Lire la ville de Jacques Poulin avec l'œil d'Italo Calvino, thèse de doctorat, Paris, Université Paris IV-Sorbonne, 2010, $474 \mathrm{f}$.

+ CUMMINS, S. S., J. Poulin, a Study of His Fictional Work, 1967-1990, thèse de doctorat, Londres (Royaume-Uni), Queen Mary and Westfield College, 1997, s. p.

+ DERAÎCHE, Myra, La parole romanesque dans La tournée d'automne de Jacques Poulin, mémoire de maîtrise, Québec, Université Laval, 2002, 140 f.

+ DUBÉ, Cécile, "Le blues de Schubert», récit. Écrire dans la maison du miroir, une expérience d'écriture. Réflexion critique: Le chat-personnage romanesque, figure métonymique, thèse de doctorat, Québec, Université Laval, 1997, 272 f.

+ DUPONT, Nathalie, L'altérité, témoin du postcolonialisme chez Jacques Poulin, mémoire de maîtrise, Sherbrooke, Université de Sherbrooke, 2001, $114 \mathrm{f}$.

+ DUPUIS, Gilles, Jacques Poulin: une dynamique de l'écriture, mémoire de maîtrise, Ottawa, Université d'Ottawa, 1990, $119 \mathrm{f}$.

+ DUSSAULT-FRENETTE, Catherine, L'expression du désir féminin adolescent. Étude des (re)configurations des normes sexuelles genrées dans quatre romans québécois contemporains, mémoire de maîtrise, Sherbrooke, Université de Sherbrooke, 2012, 153 f.

+ FORGUES, Valérie, Adèle encore une fois, roman; suivi de Relations fraternelles et quête identitaire dans trois romans québécois contemporains: L'enfant migrateur (Aude), Après la nuit rouge (Christiane Frenette), Les yeux bleus de Mistassini (Jacques Poulin), mémoire de maîtrise, Québec, Université Laval, 2010, 130 f. 
+ GRANDMANGIN, Nicolas, Jacques Poulin blues: les logiques intratextuelles, mémoire de maîtrise, Sherbrooke, Université de Sherbrooke, 1993, $171 \mathrm{f}$.

+ GREENBERG, David S., Catalysts of Closure, thèse de doctorat, Tallahassee, Florida State University, 1997, $116 \mathrm{f}$.

+ KALLA, Patrice, La littérature québécoise contemporaine: corpus limité à Volkswagen Blues de Jacques Poulin, Québec/Amérique, 1984, Babel, 1998, mémoire de maîtrise, Paris, Université Paris XII-Val-de-Marne, 2004, $86 \mathrm{f}$.

+ LALIBERTÉ, Alexandre, La lecture en miroir. Narcissisme et effets d'inconscient dans Le vieux Chagrin et Chat sauvage de Jacques Poulin, mémoire de maîtrise, Québec, Université Laval, 2013, $128 \mathrm{f}$.

+ LAVOIE, Carlo, Discours sportif et roman québécois: figures d'un chasseur du territoire, thèse de doctorat, London (Ontario), University of Western Ontario, 2002, $260 \mathrm{f}$.

+ LAVOIE, Luc, L'intertextualité dans Chat sauvage de Jacques Poulin à la lumière de la question du père, mémoire de maîtrise, Chicoutimi, Université du Québec à Chicoutimi, 2006, 118 f.

+ LEDOUX, Nathaly, La représentation de l'écrivain dans l'œuvre de Jacques Poulin, mémoire de maîtrise, Montréal, Université McGill, 1995, 125 f.

+ LEVASSEUR, Jean, Jacques Poulin. Étude thématique et narratologique de son œuvre (19671988), thèse de doctorat, Bordeaux, Université de Bordeaux III, 1989, 651 f.

+ MACFARLANE, Heather, Road Work: Theorizing the Road Trip Narrative in Anglophone, Quebecois and Indigenous Literatures in Canada, thèse de doctorat, Toronto, Université de Toronto, 2007, $219 \mathrm{f}$.

+ MARTEL, Kareen, L'intratextualité dans Les yeux bleus de Mistassini de Jacques Poulin, mémoire de maîtrise, Ottawa, Université d'Ottawa, 2006, $100 \mathrm{f}$.

+ MARTIN, Paul William, The Pencil of the Sun: Photography in Contemporary Canadian Fiction, mémoire de maîtrise, London (Ontario), University of Western Ontario, 1995, 123 f.

+ MARTINEAU, Lyne, "L'aventure au pays des fenêtres», suivi de De la sève vers le sens. Esthétique et éthique chez Jacques Poulin, Henri Lopes et Assia Djebar, thèse de doctorat, Québec, Université Laval, 2005, 293 f.

+ MÉNARD, Jean-Sébastien, Une certaine Amérique à lire: la Beat Generation et la littérature québécoise, thèse de doctorat, Montréal, Université McGill, 2007, 404 f.

+ MIRAGLIA, Anne Marie, L'écriture de l'Autre chez Jacques Poulin, thèse de doctorat, Toronto, Université de Toronto, 1990, $352 \mathrm{f}$.

+ MONTPETIT, André, Le discours sur l'écriture dans l'œeuvre romanesque de Jacques Poulin, mémoire de maîtrise, Montréal, Université du Québec à Montréal, 1995, 100 f.

+ MOORE, Monica Leigh-Anne, Coming and Going: The Effects of Displacement in Novels by Atwood, Poulin, Robin, Urquhart, mémoire de maîtrise, London (Ontario), University of Western Ontario, 1999, $119 \mathrm{f}$.

+ MORENCY, Jean, Le mythe de l'Amérique dans le roman québécois et le roman états-unien: vers une définition d'un modèle mythique, thèse de doctorat, Québec, Université Laval, 1991, $344 \mathrm{f}$.

+ NAUCHE, Bénédicte, Les personnages féminins dans l'œuvre de Jacques Poulin, mémoire de maîtrise, Montréal, Université de Montréal, 1994, 104 f.

+ NAVARRO PARDIÑAS, Blanca, La représentation de la lecture chez Jacques Poulin, thèse de doctorat, Montréal, Université McGill, 1992, 292 f.

+ O'BRIEN, Audrey, Mythic Space in the Western World: A Study of Spatial Representation in French Novels of the Fin(s) de Siècle, thèse de doctorat, Edmonton, University of Alberta, 2000, $226 \mathrm{f}$. 
+ PILOTE, Brigitte, Représentation de l'identité masculine dans deux romans québécois: Le fou du père de Robert Lalonde et Le vieux Chagrin de Jacques Poulin, mémoire de maîtrise, Montréal, Université du Québec à Montréal, 1994, 123 f.

+ QUIGLEY, Theresia Maria, The Evolution of the Child Protagonist in the Quebec and EnglishCanadian Adult Novel, thèse de doctorat, Sherbrooke, Université de Sherbrooke, 1990, $274 \mathrm{f}$.

+ RICHARD, Chantal, L'hétérolinguisme littéraire dans le roman francophone en Amérique du Nord à la fin du Xx siècle, thèse de doctorat, Moncton, Université de Moncton, 2004, $479 \mathrm{f}$.

+ RICHARDSON, Rébecca A. S., Gabrielle Roy dans l'univers de Jacques Poulin, mémoire de maîtrise, Montréal, Université McGill, 2006, 126 f.

+ ROBERTS, Paula Ann, La dualité dans l'œuvre de Jacques Poulin, thèse de doctorat, Toronto, Université de Toronto, 1997, 285 f.

+ ROTHENBURGER, Sunnie Maye Justine, Nation Creation, Nation Mutation: Hybridity in Wacousta and Volkswagen Blues, mémoire de maîtrise, Halifax, Dalhousie University, 2001, $177 \mathrm{f}$.

+ SAPP, Robert Austin, Family Narratives and the Transmission of Heritage in Transcultural Novels, thèse de doctorat, Chapel Hill, The University of North Carolina at Chapel Hill, 2013, $207 \mathrm{f}$.

+ SEGRELLES, Valérie, La dimension indienne dans Volkswagen Blues de Jacques Poulin: un exemple du roman québécois contemporain, mémoire de maîtrise, Marseille, Université de Provence-Aix-Marseille 1, 1998, $130 \mathrm{f}$.

+ SING, Pamela V., Le village québécois: idéologie et imaginaire, thèse de doctorat, Montréal, École Polytechnique de Montréal, 1993, $350 \mathrm{f}$.

+ TARDIF, Véronic, Le hockey dans l'imaginaire romanesque de Roch Carrier et de Jacques Poulin, mémoire de maîtrise, Ottawa, Université d'Ottawa, 2005, $114 \mathrm{f}$.

+ TREMBLAY, Ariane, Narration du sensible et représentation du littéraire dans Le vieux Chagrin, La tournée d'automne et Les yeux bleus de Mistassini, romans de Jacques Poulin, mémoire de maîtrise, Québec, Université Laval, 2012, 125 f.

+ VACHON, Karine, La transfictionnalité dans l'œuvre de Jacques Poulin, mémoire de maîtrise, Chicoutimi, Université du Québec à Chicoutimi, 2009, 105 f.

+ VILLARD, Victoria, Le héros à la recherche de lui-même dans quatre romans de Jacques Poulin: Les grandes marées (1979) [sic], Volkswagen Blues (1984), Le vieux Chagrin (1989), La tournée d'automne (1993). Une approche littéraire, mémoire de maîtrise, Lyon, Université Jean-MoulinLyon III, 2003, $113 \mathrm{f}$.

+ WEISMAN, Adam Paul, Postcolonialism in North America: Imaginative Colonization in Henry Thoreau's A Yankee in Canada and Jacques Poulin's Volkswagen Blues, thèse de doctorat, Cambridge, Harvard University, 1995, 263 f.

+ ZAHND, Elizabeth A., Images of the United States in Contemporary Narratives of Quebec and the Francophone Caribbean, thèse de doctorat, Champaign, University of Illinois at UrbanaChampaign, 1999, $150 \mathrm{f}$.

\section{F. A u tres}

+ BEAULIEU, Ivanhoé, «Petit collage (intime et fictif) au sujet d'un écrivain de Québec-la-ville où il est question de Guy Lafleur», Nord, n 2, hiver 1972, p. 71-72.

+ DÉSY, Jean, « Jean Désy à Jacques Poulin, lettre à un écrivain vivant», Mœbius, no 131, novembre 2011, p. 113-116.

+ HOMEL, D., «Spring Tides; Jacques Poulin», Essays on Canadian Writing, n 36, 1988, p. 87-89. 
+ LALONDE, Jeannine, «La nostalgie de Jacques Poulin", Brèves littéraires, n 77, 2008, p. 74.

+ MARTEL, Réginald, «Salut, frère Jacques», Nord, nº 2, hiver 1972, p. 69-70.

+ MORENCY, Pierre, «Le plus grand menteur de la ville de Québec ou Lettres à Jacques Poulin», Nord, $\mathrm{n}^{\circ}$ 2, hiver 1972, p. 48-58.

+ MORENCY, Jean, «Jacques Poulin. Partir pour le pôle intérieur de soi-même», Nuit blanche, $n^{\circ} 45$, septembre-octobre-novembre 1991, p. 36-39.

+ OUELLET, Geneviève, "Jacques Poulin, Québec, comme une empreinte au cœur», Québec français, n 151, automne 2008, p. 33-36. 